\title{
What is the minimal systemic risk in financial exposure networks?
}

\author{
Christian Diem ${ }^{\mathrm{a}, \mathrm{b}, \mathrm{e}}$, Anton Pichler ${ }^{\mathrm{b}, \mathrm{c}, \mathrm{d}}$, Stefan Thurner ${ }^{\mathrm{b}, \mathrm{e}, \mathrm{f}, \mathrm{g}, *}$ \\ a Institute for Statistics and Mathematics, WU Vienna University of Economics and Business, Welthandelsplatz 1, A-1020, Austria \\ ${ }^{\mathrm{b}}$ Complexity Science Hub Vienna, Josefstädter Straße 39, A-1080, Austria \\ ${ }^{\mathrm{c}}$ Institute for New Economic Thinking, University of Oxford, Manor Road, OX1 3UQ UK \\ ${ }^{\mathrm{d}}$ Mathematical Institute, University of Oxford, Woodstock Road, Oxford OX1 3LP, UK \\ e IIASA, Schlossplatz 1, Laxenburg A-2361, Austria \\ ${ }^{\mathrm{f}}$ Section for Science of Complex Systems, Medical University of Vienna, Spitalgasse 23, A-1090, Austria \\ ${ }^{g}$ Santa Fe Institute, 1399 Hyde Park Road, Santa Fe, 87501 NM, USA
}

\section{A R T I C L E I N F O}

\section{Article history:}

Received 15 May 2019

Revised 10 February 2020

Accepted 8 March 2020

Available online 12 April 2020

\section{Keywords:}

Systemic risk-efficiency

Interbank market

Financial networks

Contagion

Network optimization

Mixed-integer linear programming

DebtRank

Network topology measures and Systemic risk

\begin{abstract}
A B S T R A C T
We quantify how much systemic risk can be eliminated in financial contract networks by rearranging their network topology. By using mixed integer linear programming, financial linkages are optimally organized, whereas the overall economic conditions of banks, such as capital buffers, total interbank assets and liabilities, and average risk-weighted exposure remain unchanged. We apply the new optimization procedure to 10 snapshots of the Austrian interbank market where we focus on the largest 70 banks covering $71 \%$ of the market volume. The optimization reduces systemic risk (measured in DebtRank) by about $70 \%$, showing the huge potential that changing the network structure has on the mitigation of financial contagion. Existing capital levels would need to be scaled up by a factor of 3.3 to obtain similar levels of DebtRank. These findings underline the importance of macroprudential rules that focus on the structure of financial networks. The new optimization procedure allows us to benchmark actual networks to networks with minimal systemic risk. We find that simple topological measures, like link density, degree assortativity, or clustering coefficient, fail to explain the large differences in systemic risk between actual and optimal networks. We find that if the most systemically relevant banks are tightly connected, overall systemic risk is higher than if they are unconnected.
\end{abstract}

(c) 2020 The Author(s). Published by Elsevier B.V. This is an open access article under the CC BY-NC-ND license. (http://creativecommons.org/licenses/by-nc-nd/4.0/)

\section{Introduction}

Increasing capital requirements for market participants is an obvious suggestion for improving the resilience of financial systems and, in particular, for reducing systemic risk in financial markets. Examples of innovative policy proposals, where capital requirements depend on macroprudential regulation are Cont et al. (2010), who propose capital requirements in relation to the Contagion Index values of banks, Gauthier et al. (2012), who suggest that bank capital buffers should correspond to their contributions to overall systemic risk, Markose (2012), who proposes a capital surcharge related to the eigenvector

\footnotetext{
* Corresponding author at: Section for Science of Complex Systems, Medical University of Vienna, Spitalgasse 23, A-1090, Austria.

E-mail addresses: christian.diem@s.wu.ac.at (C. Diem), anton.pichler@maths.ox.ac.uk (A. Pichler), stefan.thurner@muv.ac.at (S. Thurner).
} 
centrality of banks in the financial network, and Alter et al. (2015), who show that capital requirements based on eigenvector centrality can save up to $15 \%$ of total system losses. Moreover, in the classical risk measure literature, following Artzner et al. (1999) and Föllmer and Schied (2002), the risk of an asset is measured by the amount of capital that needs to be added to the position in order to make the position acceptable to the regulator or to the firm itself. This approach can be extended to determine the capital requirements for financial institutions to bring systemic risk to levels that are acceptable to the regulator; see for example, Feinstein et al. (2017) or Biagini et al. (2018).

In the recent past, after the last financial crisis, bank capital requirements have been adjusted upwards. In the Basel III Accord the regulatory minimum capital requirements for Common Equity Tier 1 (CET1) have been increased from 2\% to 4.5\%, and Tier 1 Capital from 4\% to 6\% (BCBS, 2011a). Additionally, a capital conservation buffer has been introduced by increasing CET1 and Tier 1 capital further to $7 \%$ and $8.5 \%$, respectively. On top of this, national authorities can set an additional countercyclical buffer in the range between zero and $2.5 \%$ for phases of excessive credit growth. Global systemically important institutions have to meet additional CET1 requirements in a range of $1-2.5 \%$ (BCBS, 2011c).

Bank capital levels have been steadily increasing since the introduction of these new regulations. The monitoring report of the Basel Committee (BCBS, 2011b) shows that for a sample of 86 international banks with Tier 1 Capital larger than \$3bn, the CET1 increased from 7.2\% to 12.7\% in the period from 2011 to 2018 (BCBS, 2011b, Graph 15). For Germany, Spain, France, and Italy, ECB data shows increases in Tier 1 capital ratios from $9.2 \%, 8.1 \%, 8.4 \%$ and $6.9 \%$ to $16.4 \%, 13.2 \%, 15.3 \%$, and $14.4 \%$, respectively, for the period from 2008 to $2017^{1}$. Nonetheless, some indicators of systemic risk suggest that systemic risk levels are not declining, but are still substantially higher than before the financial crises. A prominent example is the SRISK indicator of Brownlees and Engle (2016), which shows that the systemic risk level in Europe now is twice as high as it was before the crises $^{2}$.

However, capital levels for absorbing shocks are only one part of the story in the context of systemic risk. The other essential component that determines systemic risk is the exposure network that is generated by contracts between financial agents. In particular, these networks capture the risks of potential cascading events that could threaten large fractions of financial markets with failure. This fact is reflected in a number of works such as in Allen and Gale (2000), Freixas et al. (2000), Eisenberg and Noe (2001), Boss et al. (2004a), Cont et al. (2010), Gai and Kapadia (2010), Battiston et al. (2012c), Markose et al. (2012) Thurner and Poledna (2013) and Glasserman and Young (2015).

It is therefore natural to ask what contributions to systemic risk originate specifically from networks and how their topology influences systemic risk. Indeed, many contributions to the systemic risk literature investigate the effect of network characteristics on systemic risk. Allen and Gale (2000) compare the effects of different network topologies, such as rings, fully connected graphs, and interconnected subgroups on interbank market stability. In Boss et al. (2004a) the role of scale- free network topologies in the context of systemic risk and stability is discussed. In Boss et al. (2004b) the betweenness centrality measure is introduced as a network-based measure for systemic risk. Nier et al. (2007) investigate the effects of network connectivity and concentration on contagious defaults. Gai and Kapadia (2010) employ a stylized analytical contagion model and look at the fraction of defaulting banks for given average degrees. Puhr et al. (2012) employ panel regressions to study the effects of network measures like Katz centrality on the number of defaulting banks, which are obtained from a simulation study. The concept of too interconnected to fail is also part of this discussion and is investigated, for example, by Markose et al. (2012). Glasserman and Young (2016) dedicate a considerable part of their literature review to this topic. These and many more theoretical and empirical works indicate the possibility of using networks of financial connections as a leverage point for reducing systemic risk in a financial system as an effective alternative to costly capital requirements that have been shown to have limited effects on systemic risk reduction (Poledna et al., 2017). We also find in our current study that the reorganization of the interbank networks can yield lower levels of DebtRank than a Basel III-like equity increase. If systemic risk can be effectively reduced by altering the underlying exposure network characteristics, this should be prominently factored into financial market stability policies. It is therefore essential to systematically estimate the full potential for network-based systemic risk reduction.

In this work we propose a method for quantifying the systemic risk reduction potential in empirically observed direct exposure networks by employing standard optimization techniques. The systemic risk of a network is measured with the so-called DebtRank (Battiston et al., 2012c). The actual optimization relies on an approximation of the DebtRank, because the DebtRank is computed iteratively and is thus hard to use in optimization problems. The approximation is based on the direct impacts of defaulting banks on their neighboring nodes in the exposure network. We show how the systemic risk optimization can be solved as a mixed integer linear program (MILP) by standard reformulation techniques. The optimization problem can be solved by state-of-the-art optimization algorithms and could therefore also be easily implemented in practice. In the empirical part of this study we show the effectiveness of the proposed method by applying it to a data set containing ten quarterly observations of the Austrian interbank liability network from 2006 to 2008. Our findings for the 70 largest banks suggest that the DebtRank of individual banks can be reduced on average by a factor of 3.5 or $71 \%$. This means sizeable reductions of the DebtRank for almost all of the 70 banks across the ten quarters with only a few exceptions. We evaluate the effectiveness of our optimization by calculating that bank equity would need to be increased by factors of between 2.38 and 4.26 to achieve the same level of DebtRank in the respective quarter. The average scaling factor is 3.32 ,

\footnotetext{
1 ECB Statistical Data Warehouse: Consolidated banking data set.

2 SRISK levels for different regions are provided by https://vlab.stern.nyu.edu/welcome/risk/
} 
thus on average $232 \%$ of the existing bank capital would need to be added to the banking system to reduce the DebtRank of the empirical networks to the level of the optimized networks. For comparison, the increase in Tier1 capital from $4 \%$ to $8.5 \%$ by Basel III corresponds to a scaling factor of 2.125. In comparison to this Basel III scenario, the optimization still reduces DebtRank by a factor of 1.61 .

In practice, due to the current lack of incentive schemes for systemic risk management Leduc and Thurner (2017), financial networks do not evolve toward systemically optimal configurations, and obviously they do not result in any way from such optimization procedures. However, our study can give an estimate for the systemic risk reduction potential stemming from a specific reorganization of empirically observed networks. The same optimization algorithm can be used to compute network configurations that yield a maximum of overall systemic risk. In this way, for any observed financial network, the proposed optimization procedure yields a "range" of network structures, corresponding to minimal and maximum DebtRank. This allows us to identify network characteristics that are typical for low, medium, and high DebtRank.

Closely related studies include Poledna and Thurner (2016) and Leduc and Thurner (2017), which investigate how systemic risk can be reduced by changing the underlying networks when financial agents are incentivized to favor transactions with low systemic risk in the network. The idea of applying network optimization techniques that are commonly used in operations research to systemic risk reduction is relatively new. It has been pioneered in the specific context of overlapping portfolio and fire sales by Pichler et al. (2020) who find reductions in systemic risk of around 50\% by rearranging the network structure of the 49 major european banks' government bond portfolios. However, the optimization approach there - a quadratically constrained quadratic program (QCQP) - is substantially different from the one presented here. A recent paper by Krause et al. (2019) focuses on small homogeneous macroeconomic shocks affecting the assets of all banks simultaneously and how these shocks are amplified in the banking system. They show a Monte Carlo algorithm for finding minimal and maximal networks with respect to the amplification of such small homogeneous macro shocks. Another related study is Aldasoro et al. (2017). The authors employ a theoretical model of the interbank network where optimizing risk-averse banks invest in illiquid assets and lend to each other. In their model they account for contagion originating from liquidity hoarding, interbank interlinkages, and fire sales. Their model leads to a specific interbank network for which properties of the network topology are reported.

The paper is organized as follows. Section 2 presents our approach to quantifying systemic risk. In Section 3 we derive the optimization problem for reducing DebtRank. We discuss the data and the results of the application to the Austrian interbank market in detail in Section 4. We conclude in Section 5.

\section{Quantifying systemic risk}

Quantification of systemic risk in financial networks is a non-trivial task and depends on specific aspects of interest. Based on very different ideas, various systemic risk measures have been suggested. Some, such as those based on networks, have already been mentioned above. Other well-known approaches include the $\Delta$ CoVaR, by Adrian and Brunnermeier (2016), which measures the tail dependence of bank asset returns, the systemic expected shortfall (SES) by Acharya et al. (2017) measuring the tendency of a bank to be undercapitalized if the whole system is undercapitalized, the SRISK measure proposed by Brownlees and Engle (2016), and the put option portfolio approach by Lehar (2005). The advantage of these market-based measures for systemic risk measurement is that they do not require detailed (often restricted) information from financial networks but estimate systemic risk from openly accessible data. These models are unable to estimate the contributions from cascading effects through financial exposure networks. The difference between these two strands of literature is emphasized by Benoit et al. (2017).

Here we choose the network-based measure DebtRank as a way to quantify systemic risk. The following method for minimizing systemic risk is then applicable to all direct financial exposure networks, whenever DebtRank is used as the measure for systemic risk. Examples of analyzing systemic risk on networks include interbank networks Battiston et al. (2012c); Thurner and Poledna (2013), derivatives and foreign exchange Poledna et al. (2015), and credit-default swaps Leduc et al. (2017). Without loss of generality for any kind of direct exposure network, we demonstrate the method for interbank asset-liability networks.

We model the interbank market with $N$ banks as a directed weighted network represented by the asset-liability matrix, $L$. The nodes represent banks; links are the liabilities between banks. If bank $j$ lends $L_{i j}$ (monetary units) to bank $i$, we represent this as a directed link from node $i$ to node $j$ with a corresponding weight of $L_{i j}$. $L_{i j}$ is $j$ 's exposure towards $i$, (i.e., if $i$ defaults the amount $L_{i j}$ is at risk for $j$ ). We denote the total interbank liabilities of bank $i$ to all others in the network by $l_{i}=\sum_{j=1}^{N} L_{i j}$; the sum of all loans from $i$ to other banks is $a_{i}=\sum_{j=1}^{N} L_{j i}$. The equity of bank $i$ is denoted by $e_{i}$, and the total interbank market volume in the network is $\bar{L}=\sum_{i=1}^{N} l_{i}=\sum_{i=1}^{N} a_{i}$. The relative economic weight of bank $i$ in the network is $v_{i}=a_{i} / \bar{L}$.

In the case of the default of $i$, we assume that bank $j$ needs to write off $L_{i j}$ of its assets. For simplicity, we assume zero recovery. Note that this assumption is not entirely unrealistic for short time scales and is frequently used in the literature. In general, a simple way of assuming a positive recovery rate is to assume that the exposure at default is simply reduced by this recovery rate. If the recovery rate is assumed to be, say, $40 \%$ on the liability $L_{i j}$, this means that at most $0.6 L_{i j}$ has to be written off by bank $j$, if bank $i$ defaults (loss given default). If we assume a general recovery of $x \in[0,1]$ this would amount to using the weighted network $(1-x) L$ for the DebtRank calculations. Due to the nature of Eq. (1) this is the same 
as considering a scaled equity vector $(1 /(1-x)) e$, and this case is treated in Appendix C. Thus, the assumption of a recovery rate would not affect the interpretation of our results qualitatively.

As a bank cannot have negative equity, the maximum impact that $i$ can have on $j$ is $e_{j}$. This motivates the definition of the direct impact matrix,

$$
W_{i j}=\min \left(\frac{L_{i j}}{e_{j}}, 1\right),
$$

which denotes the share of $j$ 's equity lost due to the default of bank $i$. As stated above, we quantify systemic risk by using DebtRank. DebtRank is a recursive centrality measure designed specifically for networks of direct financial exposures and quantifies the impact of bank $i$ on the entire network if $i$ defaults. Every bank $i$ has a DebtRank value, $R_{i}$, between zero and one; $R_{i}=0$ means that bank $i$ has no impact on other banks, whereas $R_{i}=1$ indicates that the entire interbank assetweighted equity of the system is at risk, should $i$ default ${ }^{3}$ In that sense, $R_{i}$ is the fraction of the affected total value in the network through $i$ 's default.

Definition 1 (DebtRank). DebtRank is defined by an iterative procedure that involves two state variables, $h$ and $s$. $h_{i}(t)$ measures the level of distress at iteration $t$; it is the fraction of equity $\left(e_{i}\right)$, lost due to losses that occurred before time $t$. Consequently, $h_{i}(t) \in[0,1]$, where $h_{i}(t)=1$ means default; i.e. $100 \%$ of bank is equity is lost. The variable $s_{j}(t) \in\{U, D, I\}$ takes one of three states: undistressed, distressed, and inactive. Define $S$ as the set of banks that are distressed at initial time $t=1$. Then, variables are initialized with $h_{i}(1)=\psi \forall i \in S$ and $h_{i}(1)=0 \forall i \notin S$, and $s_{i}(1)=D \forall i \in S$, and $s_{i}(1)=U \forall i \notin S$. The variable $\psi$ specifies the amount of initial distress where $\psi=1$ means default. For $t \geq 2$ first $h_{i}(t)$ is updated simultaneously for all $i$, followed by an update of $s_{i}(t)$, for all $i$. The update recursion is given by

$$
h_{i}(t)=\min \left(1, h_{i}(t-1)+\sum_{j} W_{j i} h_{j}(t-1)\right) \text {, where } j \in\left\{j: s_{j}(t-1)=D\right\},
$$

and

$$
s_{i}(t)= \begin{cases}D & \text { if } h_{i}(t)>0 ; s_{i}(t-1) \neq I \\ I & \text { if } s_{i}(t-1)=D \\ s_{i}(t-1) & \text { otherwise. }\end{cases}
$$

The iterative procedure ends after $T$ steps at which all nodes are either undistressed or inactive. The DebtRank of bank $i$ is defined as

$$
R_{i}=\sum_{j=1}^{N} h_{j}(T) v_{j}-\sum_{j=1}^{N} h_{j}(1) v_{j}=\sum_{j \neq i} h_{j}(T) v_{j}
$$

The last equality holds because we assume that only bank $i$ initially defaults, leading to $h_{i}(1)=h_{i}(T)=1$. The initial distress is set to $\psi=1$, and the initial default set contains only bank $i$. The share of bank $j$ 's interbank assets, $v_{j}$, can be interpreted as the relative economic value of $j$ in the network. When bank $i$ initially defaults, and as a consequence bank $j$ loses a fraction of its equity, $h_{j}(T)>0$. Then, the value $v_{j}$ determines how strong this loss is reflected in the DebtRank of bank $R_{i}$. We define the systemic risk of the entire market as the sum of the individual bank DebtRanks, i.e.

$$
R=\sum_{i=1}^{N} R_{i}
$$

For a motivation of this definition, see also Poledna and Thurner (2016). For comparative purposes, we also employ a variation of this definition of DebtRank presented in Bardoscia et al. (2015). We refer to this definition as DebtRank2. For more details, see Appendix G. DebtRank2 has been suggested as a micro foundation for shock propagation in networks and is directly derived from bank balance sheet identities. Bardoscia et al. (2015) acknowledge that the original DebtRank formulation can lead to underestimations of systemic risk because shocks propagate through a node only for a single time and subsequently the node becomes inactive. If a bank receives shocks from different neighbors at sequential times it transmits only the first shock, as it becomes inactive after receiving the first shock. Similarly, when a bank receiving a shock is part of a loop and will receive another shock from the same loop at a later time, it will not forward the shock a second time. The two DebtRanks are the same for tree networks and some other special structures. In general, DebtRank is a lower bound to DebtRank2 (Bardoscia et al., 2015). However, since DebtRank2 allows for multiple shock transmissions of a node this leads (in principle) to an infinite number of shocks on networks that contain loops. In practice, the algorithm stops when the shocks become smaller than a predefined value $\epsilon$. However, in the original DebtRank formulation Battiston et al. (2012c) point out that an infinite cycling of shocks when loops are present might not be desirable. For this reason and because in the literature the original DebtRank is more widely used, we stick to the original DebtRank for the rest of the paper. Another interesting generalization of DebtRank is studied by Bardoscia et al. (2016), which relaxes the assumption that shocks propagate linearly.

\footnotetext{
${ }^{3}$ From the definition of DebtRank, it is obvious that $R_{i}=1$ can occur only if the weight $v_{i}=0$. Thus, in most cases $R_{i}$ is strictly smaller than one.
} 


\section{Minimizing systemic risk as an optimization problem}

This section proposes an optimization procedure ${ }^{4}$ that rewires a given interbank network to obtain a second (optimal) network that is close to the optimal DebtRank for the prevailing economic environment (i.e., for a given level of equity, bank lending and borrowing, and banks' risk exposure to other banks). Because of its recursive definition in Eq. (4), DebtRank is not representable in closed form. This makes it unpractical to use as an actual objective function of an optimization problem. Even though an optimization with respect to DebtRank is of course possible in principle, it would be computationally costly or even infeasible for large networks. Instead we propose a practical and easy- to-implement method that is capable of reducing systemic risk (DebtRank) substantially in empirical networks. For this, we approximate DebtRank, $R$, by a sum of piecewise linear concave functions that serves as objective function in the optimization.

Definition 2 (Direct Impact). The direct impact $I_{i}$ of bank $i$ on its neighbouring banks is defined by

$$
I_{i}=\sum_{j=1}^{N} W_{i j} v_{j}=\frac{1}{\bar{L}} \sum_{j=1}^{N} \min \left(\frac{L_{i j}}{e_{j}}, 1\right) a_{j} .
$$

The sum of all direct impacts is $I=\sum_{i=1}^{N} I_{i}$, which can be interpreted as a first-order approximation of the DebtRank.

Direct impact, $I$, is representable in closed form, Eq. (6), that allows us to solve the optimization problem with Mixed Integer Linear Programming (MILP) techniques. The optimization rewires links in the network in an optimal way but should leave certain network characteristics untouched. To ensure an economically meaningful comparison, we keep total assets and liabilities of banks unchanged, as well as the total market volume, $\bar{L}$. Additionally, we require the interbank risk exposure to remain the same for every bank. If $\kappa_{j}$ denotes the credit risk of bank $j$, the average credit risk exposure of bank $i$ is given by $r_{i}=\sum_{j=1}^{N} L_{j i} \kappa_{j}$. We implement these requirements as constraints in the optimization and discuss their economic meaning in more detail in Section 3.1. Now the optimization problem can be formulated as

$$
\begin{aligned}
\min _{L \in\left\{M: M \in \mathbb{R}_{+}^{N \times N}, M_{i i}=0\right\}} \sum_{i=1}^{N} \sum_{j=1}^{N} \min \left(\frac{L_{i j}}{e_{j}}, 1\right) a_{j} \\
\text { subject to } \quad l_{i}=\sum_{j=1}^{N} L_{i j} \quad, \quad \forall i \\
a_{i}=\sum_{j=1}^{N} L_{j i} \quad, \quad \forall i \\
r_{i}=\sum_{j=1}^{N} L_{j i} \kappa_{j} \quad, \quad \forall i .
\end{aligned}
$$

The values for $e, l, a, v$, and $\bar{L}$ can be obtained from bank balance sheets, whereas the interbank network $L$ is usually not publicly available. Note that if the last constraint in Eq. (7) is omitted, the optimization only requires row and column sums of $L$. Thus, optimal networks can be obtained without knowing the exact empirical network. The objective function is not linear but piecewise linear and concave because of the minimum operator; the sum of concave functions is concave. In Eq. (7) we omit $\bar{L}$ because it is just a positive multiplicative constant. The result of the optimization is the optimal asset-liability matrix, $L^{*}$. A global optimum exists because of the concavity of the objective function and due to the bounded solution space $\left(L_{i j} \in\left[0, \min \left(a_{i}, l_{i}, a_{j}, l_{j}\right)\right] \forall i j\right)$. However, the optimum is not necessarily unique. We find globally optimal solutions by solving an equivalent MILP, which is derived in the following.

The optimization problem comprises $N^{2}-N$ free variables (no self-links), which turns even moderately large interbank markets into large-scale optimization problems. To solve this problem, we linearize the objective function by reformulating it as a MILP. As the minimum function is piecewise linear, one can apply standard techniques of mathematical programming to rewrite Eq. (7) as a MILP. We use the concept of special ordered sets (SOS) and, more specifically, SOS2 constraints for the linearization of the objective function. This concept dates back to Beale and Tomlin (1970) and allows us to find a global solution.

We first provide some intuition of the behavior of the objective function Eq. (7), and then explain the reformulation in detail. As all $a_{j}$ are non-negative, we can write them inside the minimum function, and a single term in the objective function in Eq. (7) reads, min $\left(\frac{a_{j}}{e_{j}} L_{i j}, a_{j}\right)$. Fig. A.6 in the appendix shows its behavior. It increases until $L_{i j}=e_{j}$ by $a_{j} / e_{j}$ and remains constant afterwards. Below, we show how to relate each entry $L_{i j}$ to a pair of variables, $\left(y_{2 k-1}, y_{2 k}\right) . y_{2 k-1}$ accounts for the part of $L_{i j}$, where the objective still increases in $L_{i j} ; y_{2 k}$ accounts for the region, where the objective function is constant (w.r.t. $L_{i j}$ ). The economic interpretation of the transition point at $L_{i j}=e_{j}$ is that the liability of bank $i$, with respect

\footnotetext{
${ }^{4}$ We provide the $\mathrm{R}$ code for the optimization procedure and an example with simulated data on https://csh.ac.at/vis/code/network_optimization/
} 
to bank $j$, is of the same size as bank $j$ 's equity. In the case of $i$ 's default, $100 \%$ of $j$ 's equity would be destroyed. However, when $L_{i j}>e_{j}, y_{2 k}=\min \left(0, L_{i j}-e_{j}\right)$ no longer affects the objective function, as more than $100 \%$ of $j$ 's equity cannot be consumed. Note that the remaining loss of $\min \left(0, L_{i j}-e_{j}\right)$ is borne by creditors of $j$, which are outside the interbank system. We now show more formally how the objective function can be transformed into a MILP with the help of the variables $y$ and a set of dummy variables, $\delta$.

We stated the optimization problem in matrix terms. In numerical optimization it is more common to optimize over vectors. We therefore rewrite $L \in \mathbb{R}_{+}^{N \times N}$ into a vector $x \in \mathbb{R}_{+}^{N^{2}}$ by stacking the columns of $L$,

$$
x=\operatorname{vec}(L)=\left(L_{11}, \ldots, L_{N 1}, L_{12} \ldots, L_{N 2}, L_{1 N}, \ldots, L_{N N}\right)^{\top} .
$$

Note that for ease of notation and implementation we keep the diagonal entries $L_{i i}, \forall i$. Similarly, we define vectors of length $N^{2}$ for representing assets, liabilities, and equities,

$$
\begin{aligned}
& \bar{e}=(\underbrace{e_{1}, \ldots, e_{1}}_{N \text { times }}, e_{2}, \ldots, \underbrace{e_{N}, \ldots, e_{N}}_{N \text { times }})^{\top}, \\
& \bar{a}=(\underbrace{a_{1}, \ldots, a_{1}}_{N \text { times }}, a_{2}, \ldots, \underbrace{a_{N}, \ldots, a_{N}}_{N \text { times }})^{\top},
\end{aligned}
$$

and

$$
\bar{l}=(\underbrace{l, \ldots, l}_{N \text { times }})^{\top} .
$$

Now we can write the objective function in Eq. (7) as

$$
\min _{x \in \mathbb{R}_{+}^{N^{2}}} \sum_{j=1}^{N^{2}} \min \left(\frac{\bar{a}_{j}}{\bar{e}_{j}} x_{j}, \bar{a}_{j}\right) .
$$

The elements in $x$ corresponding to the diagonal elements of $L$ have to be zero, which can be enforced with additional constraints or directly in the optimization software. To translate the objective function into a linear form $c^{\top} y$, every variable $x_{i}$ is split into two parts, $y_{2 i-1}$ and $y_{2 i}$, with $x_{i}=y_{2 i-1}+y_{2 i}$, and

$$
\begin{aligned}
& y_{2 i-1}=\min \left(x_{i}, \bar{e}_{i}\right), \\
& y_{2 i}=\min \left(x_{i}-\bar{e}_{i}, 0\right) .
\end{aligned}
$$

The first part, $y_{2 i-1}$, indicates the range of $x_{i}$, where an increase by $\Delta x_{i}$ leads to an increase in the objective function by $\Delta x_{i}\left(\bar{a}_{i} / \bar{e}_{i}\right)$. At $x_{i}=\bar{e}_{i}$, the objective function no longer increases with $x_{i}$. This range of $x_{i}$ is accounted for by $y_{2 i}$. To reformulate the objective function in terms of the new variables $y$, we need to introduce a vector of binary variables $\delta \in$ $\{0,1\}^{2 n^{2}}$ in the following way

$$
\delta_{j}= \begin{cases}1 & \text { if } y_{j}>0 \\ 0 & \text { if } y_{j}=0\end{cases}
$$

With $\delta$ we can formulate the following constraints for the pairs $\left(y_{2 i-1}, y_{2 i}\right)$, for all $i$,

$$
\delta_{2 i-1} \geq \delta_{2 i}
$$

$$
y_{2 i-1} \geq \delta_{2 i} \bar{e}_{i}
$$

$$
y_{2 i-1} \leq \delta_{2 i-1} \bar{e}_{i}
$$

$$
y_{2 i} \leq \delta_{2 i} \max \left(0, \min \left(\bar{a}_{i}, \bar{l}_{i}\right)-\bar{e}_{i}\right) .
$$

Constraints (15)-(18) ensure the equivalence of the reformulated problem in Eq. (20) and the original problem in Eq. (7). In particular, Eq. (15) enforces that $y_{2 i}$ can only be larger than zero if $y_{2 i-1}$ is larger than zero. Constraints (16) and (17) enforce that $y_{2 i-1}$ must be smaller than $\bar{e}_{i}$, and that if $y_{2 i}$ is bigger than zero, $y_{2 i-1}$ has to be equal to $\bar{e}_{i}$. Finally, Eq. (18) ensures that $x_{i}=y_{2 i-1}+y_{2 i}$ is smaller than the respective row and column sum of the corresponding entry in the liability matrix. We finally define the vector $c$ of length $4 N^{2}$, which determines the slope with which the respective entries in $y$ increase,

$$
c_{j}= \begin{cases}\bar{a}_{i} & \text { if } j=2 i-1, \text { and } i \leq N^{2} \\ \bar{e}_{i} & \text { if } j=2 i, \text { and } i \leq N^{2} \\ 0 & \text { if } 2 N^{2}<j \leq 4 N^{2}\end{cases}
$$


It follows that every second entry in $c_{2 i}$ is equal to zero, as the even components $y_{2 i}$ do not increase the objective function. These correspond to the part of $x_{i}$, where the objective function is capped to $\bar{a}_{i}$. The odd parts, $c_{2 i-1}$, represent the slopes. The last $2 N^{2}$ zeros ensure that the binary variables $\delta$ do not affect the value of the objective function. The objective function can now be written as $c^{\top} z$, where $z=(y, \delta) \in \mathbb{R}^{4 n^{2}}$.

The constraints for $\delta$ and $y$, Eqs. (15)-(18), are compactly reformulated as $A_{1} z=0$, where $A_{1} \in \mathbb{R}^{4 n^{2} \times 4 N^{2}}$, and 0 denotes a zero-vector of length $4 N^{2}$. The constraints on the row and column sums of the liability matrix in the initial problem, Eq. (7), can be written in standard matrix form as $A_{2} z=a, A_{3} z=l$, and $A_{4} z=r . A_{2}, A_{3}$ and $A_{4}$ are $N \times 4 N^{2}$ dimensional matrices consisting of zeros and ones ${ }^{5}$. The exact structure of the constraint matrices $A_{1}, A_{2}$, and $A_{3}$ is outlined in Appendix A. Finally, the optimization problem of Eq. (7) as a MILP reads

$$
\begin{aligned}
\min _{z \in \mathbb{R}_{+}^{4 N^{2}}} \quad c^{\top} z & \\
\text { subject to } A_{1} z & \leq 0 \\
A_{2} z & =a \\
A_{3} z & =l \\
A_{4} z & =r .
\end{aligned}
$$

This method is generic and is generally applicable to all direct financial exposure networks where systemic risk is quantified by DebtRank. If different types of financial network are considered, the liability matrix $L$ has to be replaced with the corresponding exposure matrices. Depending on the financial network type, various further constraints can be considered to ensure that certain economic properties of individual banks (which depend on the network but should kept constant) do indeed remain the same after the optimization. We continue by discussing such constraints in more detail.

\subsection{Implementing economic constraints}

As mentioned, the constraints in Eq. (7) not only ensure that banks retain their size after optimization, but they also have an important economic interpretation. Row and column sums represent the interbank liabilities and interbank assets of each bank. Keeping these constant implies that each bank retains its amount of liquidity ${ }^{6}$ from the interbank market after optimization. If we assume that the liquidity a bank requires from the interbank market originates from its operational business, it is important that this activity is not distorted by the optimization procedure.

Another important type of economic constraint is related to economic risk. In direct exposure networks, counter-party credit risk plays an important role when lending decisions are being made. In the case of indirect exposure networks, such as overlapping portfolio risk, risk associated to the financial assets held by the financial institutions play a crucial role in the making of investment decisions. The most important types are credit, market, and interest rate risk. For the sake of comparability of empirically observed reference networks and optimized networks, it is desirable to have constraints in order to ensure that the risks for the individual institutions remain comparable before and after optimization. For interbank networks none of the lending banks should end up with a higher counter-party credit risk after the optimization. To achieve this, we introduced a linear constraint to ensure that the credit risk in all interbank loan portfolios is approximately maintained. This constraint accounts for individual economic conditions of banks that are affected by the network structure. We aim to model this feature by fixing the predominant credit-risk-weighted-exposure of the interbank loan portfolio of each bank.

Let the considered credit risk indicator of bank $i$ be $\kappa_{i}$. For a given liability matrix $L$, the risk weighted interbank loan exposure of bank $j$, that is implied by the interbank network $L$, is then given by $r_{j}=\sum_{i=1}^{N} L_{i j} \kappa_{i}$, or in matrix notation, $r=L^{\top} \kappa$. The constraint in the MILP of Eq. (20) is added as

$$
A_{4} z=r \text {. }
$$

We explain in Appendix I that the formulation of constraint Eq. (21) as equality, and smaller or equal, yields the same optimal value of the problem in Eq. (20), given that the row and column sum constraints are in place. Further, this constraint also keeps the earnings from the interbank loan portfolio similar before and after optimization because the interest rate earned on an interbank loan should strongly reflect the credit risk level of the borrower. Additionally, the regulatory capital levied on the interbank loan portfolio also remains comparable because capital requirements depend on the risk-weighted assets of the respective bank. Just as the risk-weighted interbank loan exposure remains constant in the optimization, so the risk-weighted assets should also retain their size.

For the case of optimizing indirect exposure networks, similar risk constraints can be implemented. For example, Pichler et al. (2020) consider Markowitz mean-variance conditions for optimizing financial exposures emerging from common asset holdings and discuss further possible constraints. Other meaningful constraints for financial asset networks are credit risk constraints, such that the average credit risk - of, for example, a bond portfolio - remains comparable. To keep the interest

\footnotetext{
${ }^{5}$ Note that there is at least one redundant equation in this set of linear constraints, as $N$ column sums and $N-1$ row sums imply the $N$ th row sum.

${ }^{6}$ As we deal only with a single liability matrix, $L$, we implicitly assume in the optimization procedure that all liabilities have the same maturity, which is of course not realistic. If a family of matrices, $L_{1}, \ldots, L_{t}$, describing the interbank liabilities for various maturities (or maturity buckets) $1, \ldots, t$ is available, and the optimization procedure is applied to each maturity separately, so that the original maturity structure is unaffected.
} 
rate risk of fixed income portfolios similar across the optimization, another linear constraint can account for the maturity or duration of the assets. In general, different financial networks will require different economic constraints.

\section{Optimization of empirical Austrian interbank networks}

The solution to the MILP yields a network with minimal direct impacts, $I$, but not necessarily one with minimal systemic risk in terms of DebtRank, $R$. However, our computations demonstrate the great effectiveness of this approximation in massively reducing overall systemic risk.

We apply the optimization to a data set consisting of 10 snapshots of Austrian interbank networks at 10 quarters from 2006 to 2008. The Austrian interbank network has been studied before by, for example, Boss et al. (2004a), Elsinger et al. (2006), Caccioli et al. (2015) ${ }^{7}$. The sample contains between 824 and 846 banks. The Austrian banking system accommodates many very small cooperative banks, which cannot be considered as systemically important. We use the 70 largest banks with respect to total assets in the corresponding quarter for numerical feasibility. These account for about $86 \%$ of total assets; the 70th largest bank accounts for around $0.12 \%$ of total assets. The 70 banks with the largest total assets cover around $71 \%$ of the interbank market. We choose the banks' total asset size as the selection criterion because total assets should be a more stable quantity than interbank market shares. We deal with a fully anonymized data set, which makes it impossible to estimate the bank's credit risk indicators, $\kappa_{i}$. Approaches for estimating $\kappa$ are outlined in Appendix $H$ for cases where sufficient data would be available. For demonstration purposes we approximate $\kappa$ by the leverage ratio of the banks'

$$
\kappa_{i}=\frac{\text { total }_{\text {assets }}}{\text { total } \text { assets }_{i}-\text { total liabilities }} .
$$

We assume that a higher leverage ratio implies higher credit risk. To solve the optimization problem numerically, we employ the MILP solver cplex, available in the R Optimisation Infrastructure (ROI) package (Theußl et al., 2019). The optimization can be performed on a standard notebook and takes from a few minutes to several hours, depending on the network sample.

To compare the effectiveness of network optimization with actual policies we also compute the DebtRank for increased values of bank equity. As pointed out in the introduction, Tier 1 capital requirements increased under Basel III from 4\% to 8.5\%. ECB data shows increases of Tier 1 capital ratios for Germany, Spain, France, and Italy from 9.2\%, 8.1\%, 8.4\% and 6.9\% to $16.4 \%, 13.2 \%, 15.3 \%$, and $14.4 \%$, respectively, for the period from 2008 to 2017. The Basel III increase corresponds to a scaling factor of 2.215, and the increases in the ECB data range from 1.63 to $2.09^{8}$. Our sample from 2006 to 2008 falls into the time period before the Basel III regime and ends when ECB capital measurements start. Therefore, we can meaningfully compute how DebtRank levels would have looked under the Basel III regime. In our sample the mean equity to total assets ratio, $1 / \kappa_{i}$, across time and across observations is $10.20 \%$; the median is $7.19 \%$. Our baseline scenario for equity increases computes the DebtRank for the empirical networks with a hypothetical Basel III equity vector 2.125 . e. In Appendix C we show DebtRank levels for various scaling factors up to 4, and we compute the "break-even" capital increase at which the same level of DebtRank reduction is achieved as in the optimization.

\subsection{Results}

The reduction of systemic risk obtained by the optimization procedure is summarized in Fig. 1(a). The values of the total DebtRank, $R$, after optimization (blue triangles) are substantially lower than the corresponding empirical (red squares) ones across all quarters. The average DebtRank in the empirical and optimized networks is around 12.51 and 3.54 , respectively, meaning that the average total DebtRank reduction amounts to approximately $71 \%$, or a factor of roughly 3.5 . The Basel III equity increase scenario (orange) reduces average DebtRank substantially to 5.7, but is not as effective as the optimization procedure. In Appendix $C$ we find that on average the original equity would need to be increased by a factor of 3.32 to achieve the same reduction of DebtRank as the network optimization. Fig. 1(b) shows the individual DebtRanks, $R_{i}$, of the 70 banks for the empirical case (red squares) and the optimized (blue triangles). The size of the symbols represents the banks' interbank liabilities, $l_{i}$. The figure shows two facts. The first is that in the optimized network at least one bank always remains relatively systemically risky with respect to the majority of banks, even though their DebtRank is substantially reduced. The second observation is that the DebtRank reduction for small- and medium- sized banks, indicated by triangle size, seems to work even better than for the large banks. Fig. D.10 shows the relationship of DebtRank $R_{i}$ and interbank liabilities $l_{i}$ in more detail. In the empirical networks small banks severely "punch above their weight", that is, banks with small interbank liabilities frequently have high DebtRanks, $R_{i}$, and - judging by their size - their default would cause unnecessary systemic events. The optimization remedies this problem and renders banks with small interbank liabilities systemically negligible.

In Fig. 1(a) it is seen that from Q8 to Q10 the optimized DebtRank increases, while the empirical DebtRank continues its downward trend. To understand why, in Fig. 1(c) we show the total interbank market volume and the total equity in the system over time, relative to the values in Q1. Larger levels of equity - all other things kept equal - should reduce DebtRank,

\footnotetext{
7 Caccioli et al. (2015) uses the same data set which originally consisted of 12 quarters. Due to obvious data errors we dropped 2 of the 12 observations.

8 We mention here that in the DebtRank framework it does not make a difference if the increase in the capital ratio is realized by increasing equity or shrinking the asset side, since the DebtRank calculation hinges on the matrix $W$ where $W_{i j}=\min \left(1, L_{i j} / e_{j}\right)$ and obviously min $\left(1,0.5 L_{i j} / e_{j}\right)=\min \left(1, L_{i j} / 2 e_{j}\right)$.
} 

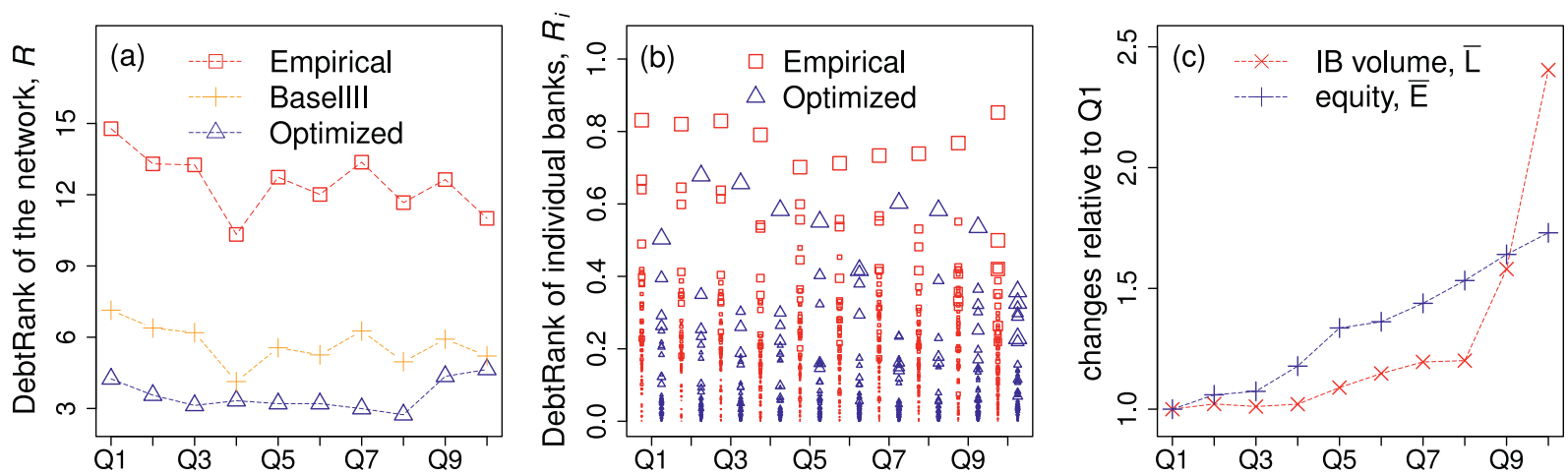

Fig. 1. (a) Total DebtRank, $R$, of the empirical Austrian interbank networks across 10 quarters from 2006 to 2008 (squares). For the optimized networks the DebtRank is drastically reduced (triangles). The optimization reduces systemic risk (measured in DebtRank) by a factor of approximately 3.5. (b) Individual DebtRank, $R_{i}$, of 70 banks for the empirical and optimized networks in the respective quarters. Here symbol sizes are proportional to the banks' interbank liabilities, $l_{i}$. We see that typically large banks have high $R_{i}$, however note that there are many exceptions, with small banks having considerable systemic risk. (c) Total interbank market volume, $\bar{L}$, and equity, $\bar{E}=\sum_{i=1}^{N} e_{i}$, over the ten quarters. While decreasing in the first eight quarters, the ratio $\bar{L} / \bar{E}$ increases substantially in Q9 and Q10.

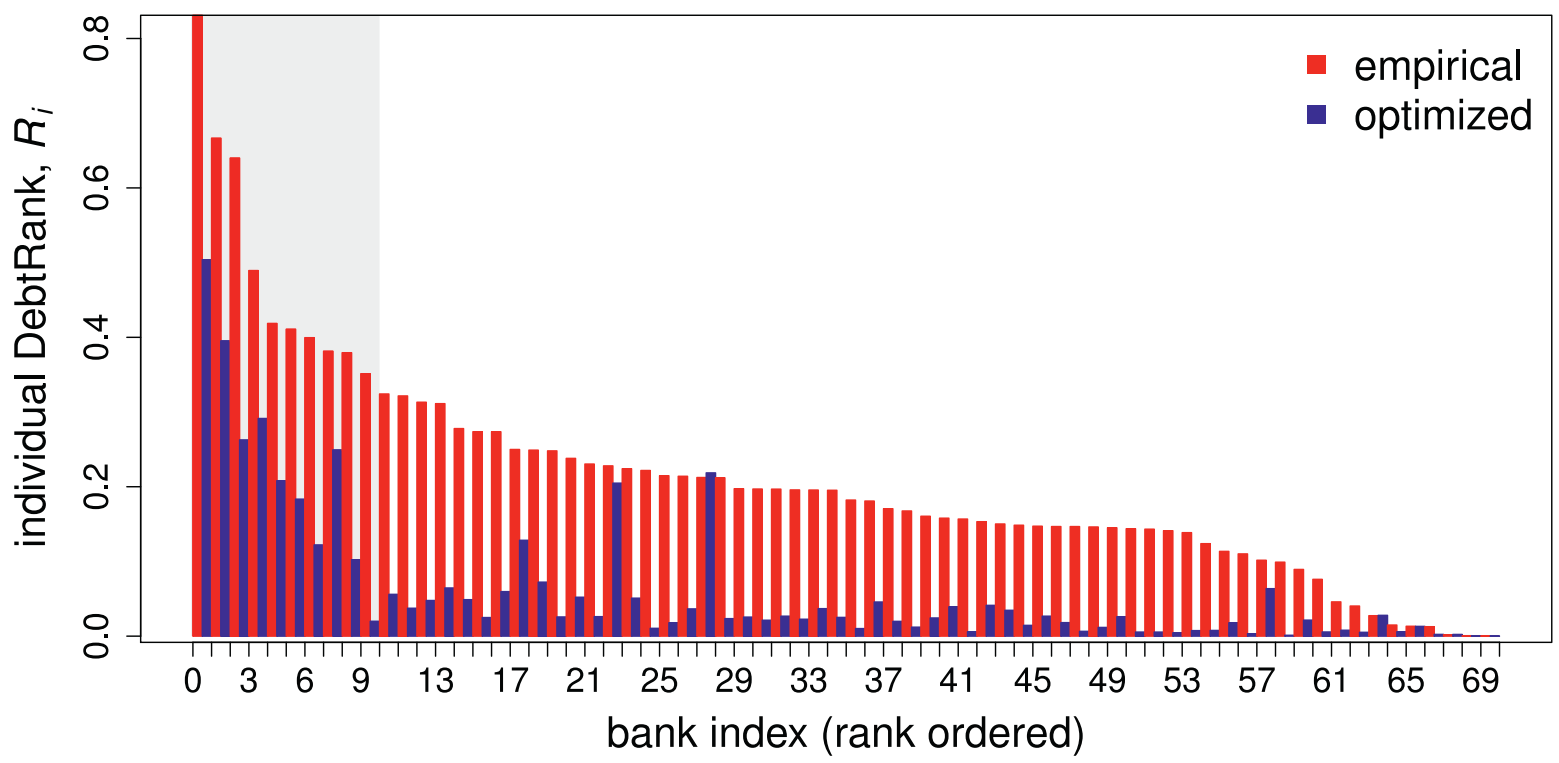

Fig. 2. Systemic risk profile (DebtRank $R_{i}$ ) of the 70 banks for the empirical (red) and the minimized (blue) networks in quarter Q1. Banks are rank-ordered with respect to their DebtRank, $R_{i}$, in the empirical network. It can be clearly seen that systemic risk is drastically reduced for practically all banks, with only one exception. For the 10 most risky banks a reduction of DebtRank, $R_{i}$ by a factor of 2.1 is observed; for higher ranks, the reduction by a factor of 5.1 is even more drastic. Similar results hold for the other quarters. (For interpretation of the references to color in this figure legend, the reader is referred to the web version of this article.)

and an increase in the market volume should increase DebtRank. Thus, the sharp increase in the market volume, $\bar{L}$, from Q8 to Q10 could be the explanation for the observed increase in the optimized DebtRank.

Fig. 2 depicts the systemic risk profile for Q1, where $R_{i}$ is shown for 70 banks of the empirical and the optimized case. Banks are ordered according to their empirical DebtRank; $R_{i}$; the most systemically risky institution is shown to the very left. The effectiveness of the optimization is clearly seen. DebtRank levels are decreased substantially for almost all 70 banks, with two exceptions, where banks have a slightly higher DebtRank after the optimization. For the 10 most risky banks (shaded gray) DebtRank is reduced by a factor of around 2.1; for higher ranks, the reduction is even more pronounced, and amounts to a factor of 5.1. For most banks, DebtRank is decreased to marginal levels. Similar observations hold true for all quarters; in some, a DebtRank reduction is achieved for all 70 banks.

Fig. 3 shows the relation of our objective function (direct impacts), I, and DebtRank, $R$, that serves as our measure of systemic risk, with which we also judge the effectiveness of the optimization. On the network level, the total DebtRank and direct impacts of the empirical networks are linearly related with a correlation coefficient of $\rho_{\mathrm{emp}}=0.81$, and a $p$ - 

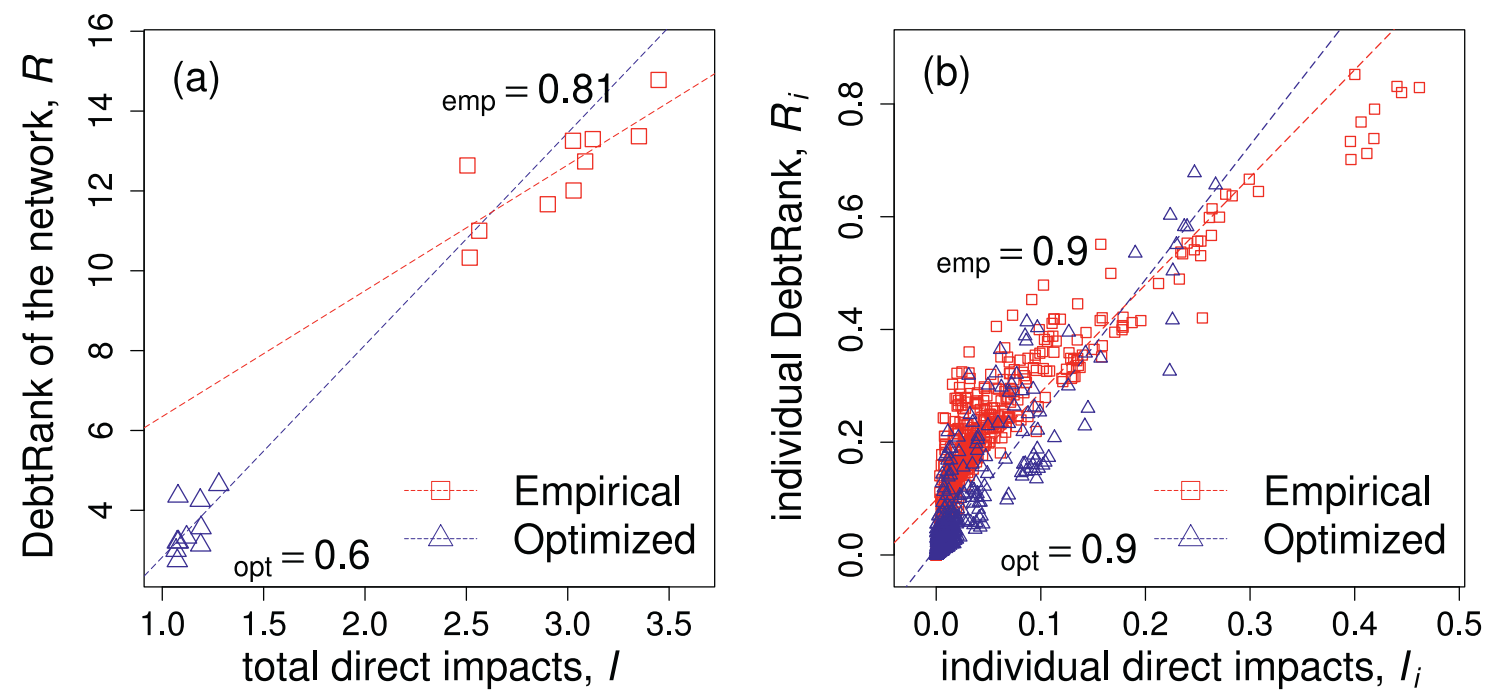

Fig. 3. (a) Total DebtRank values, $R$, versus direct impacts, $I$, for the 10 quarters of the empirical (squares) and the optimized networks (triangles). The correlation coefficients of $R$ and $I$ for the empirical and optimized cases are $\rho_{\mathrm{emp}}=0.81$, with a p-value of 0.004 , and $\rho_{\mathrm{opt}}=0.6$, with a $p$-value of 0.07 , respectively. (b) The same comparison on the individual bank level, $R_{i}$ versus $I_{i}$, with $\rho_{\mathrm{emp}}=0.90$, and $\rho_{\mathrm{opt}}=0.9$. The associated $p$-values are smaller than $2.2 \mathrm{e}-16$. The dashed lines are obtained by simple linear regression.

value of $p_{\mathrm{emp}}=0.004$. This confirms a posteriori that minimizing the direct impacts is indeed a reasonable and effective way to minimize DebtRank. In the optimized networks the linear relationship is weaker $\left(\rho_{\mathrm{opt}}=0.6\right.$, and a $p$-value of $p_{\text {opt }}=$ 0.07). This indicates that the optimization achieves a stronger reduction in direct impacts than in DebtRank. Fig. 3(b) shows the same situation for the bank's individual levels of DebtRank, $R_{i}$, and direct impacts, $I_{i}$. The linear correlations for both network types are higher $\left(\rho_{\mathrm{emp}}=\rho_{\mathrm{opt}}=0.9\right)$ and their $p$-values are below $2.2 \mathrm{e}-16$. The respective results for DebtRank2 (Bardoscia et al. (2015)) are shown in Appendix G. Here, the optimization achieves an average reduction of DebtRank2 of about $15 \%$.

\subsection{How networks change during optimization}

Fig. 4 (a) shows the original interbank asset-liability network $L$ before the optimization for quarter Q1. The case after optimization is seen in (b). The nodes represent banks; size is the banks' equity; the colors represent the DebtRank value (dark red is high, light tones are medium, dark blue is low $R_{i}$ ). There are obvious differences. We now ask how the topology of interbank networks changes due to the optimization process. The average degree of the minimized network (from the binary adjacency matrix) is $\bar{k}=3.04$ versus the empirical network $\bar{k}=38.71$. The in- and out-degree distributions for the different network types pooled together for all ten quarters are provided in Fig. D.11(a) and (b). The average in- and outstrength of the networks are unchanged, due to the constraints that keep $a_{i}$ (in-strength) and $l_{i}$ (out-strength) fixed.

The most prominent observation is that networks after the optimization become sparser. Fig. 4(c) shows that the minimized network (blue triangles) is extremely sparse with an average link density of around $4.4 \%$. Every dot represents one of the 10 quarters.

The link density of the network (connectancy) is defined as the fraction of links present in the network, $d=$ $m /(N(N-1))$, where $m$ is the number of present links and $N(N-1)$ is the number of possible links. In contrast, the empirical networks (red squares) exhibit an average link density of approximately 56\%. Note, however, that by slightly thresholding the empirical networks, link densities of about $10 \%$ are obtained; see Appendix D. One could be led to believe that high link density is related to high DebtRank. This is not necessarily true. To show this, we computed the maximum direct impact networks (where we maximize Eq. (20)), which leads to networks with substantially higher DebtRank than the empirically observed ones. Interestingly, these maximized networks (green diamonds) are also sparse, with an average link density of 11\%. The maximized and thresholded networks are visualized in Fig. D.9. Sparse networks can have low or high DebtRank. In Appendix $\mathrm{F}$ we further investigate this relationship by conducting a small-scale simulation study. The initial findings which need to be corroborated in future research - suggests that for sparse networks the variance in systemic risk is substantially higher than for ones with higher connectivity. Additionally, the relationship seems to be slightly concave where, on average, systemic risk is highest for medium levels of link density.

In Fig. 4 (d) DebtRank $R$ is plotted against the degree assortativity, which is calculated as $r=\rho(v, w)$ where $\rho(.$, .) is the sample Pearson correlation coefficient, $v, w$ are $m$ dimensional vectors, and each entry corresponds to a link $L_{i j}$. The $l$ th entry corresponding to link, $L_{i j}$ is $v_{l}=k_{i}^{\text {out }}$ and $w_{l}=k_{j}^{i n}$. Compare also Thurner et al. (2018) Eq. (4.10). 
(a)
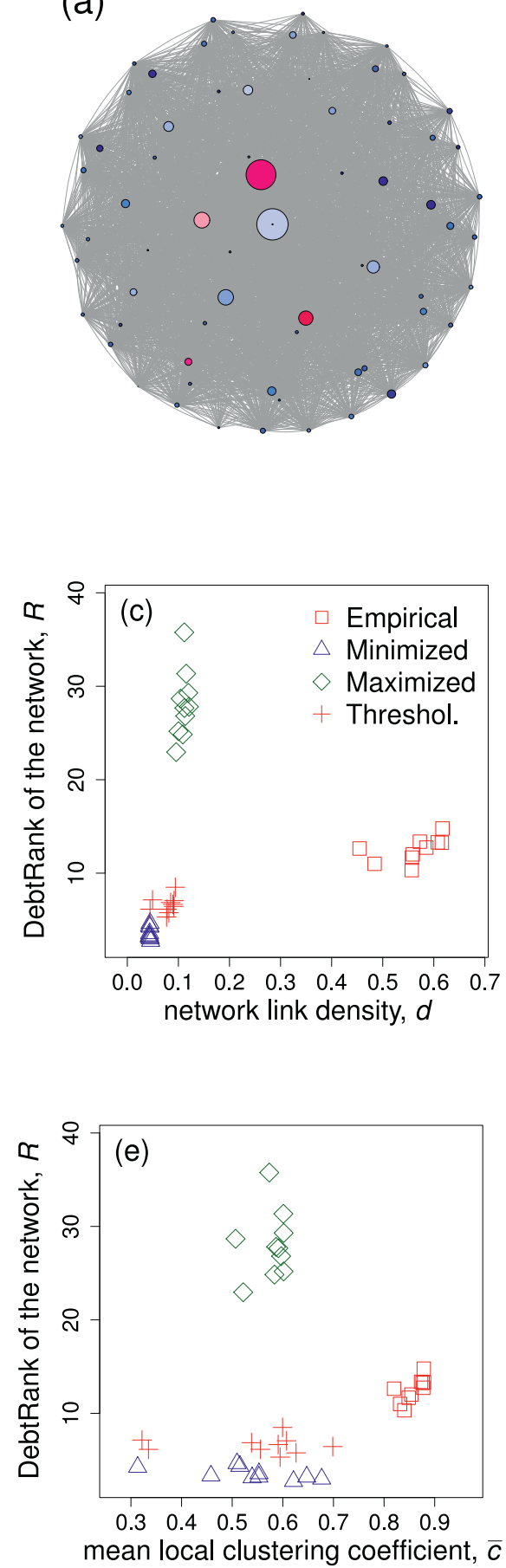

(b)
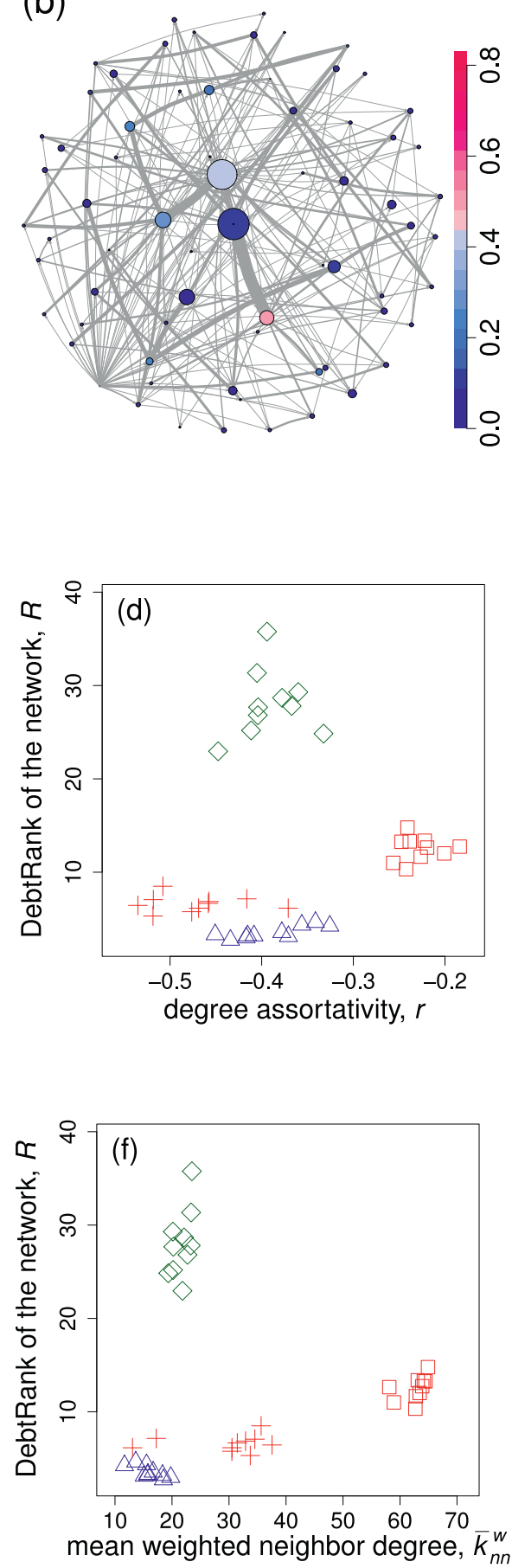

Fig. 4. Interbank networks before and after optimization. (a) Empirical asset-liability network, $L$, as in Q1, in comparison to (b) the optimized network, $L^{*}$. Node colors of banks represent their DebtRank (large DebtRank is red, small is blue). Node size is proportional to equity, $e_{i}$. It is obvious that the optimized network is considerably sparser. (c) DebtRank of the empirical, the minimized, and the maximized networks plotted against the networks' link densities, $d$. Every symbol represents a quarter. It is clearly seen that sparse networks can have both high and low DebtRank. (d) DebtRank, $R$, against the degree-weighted assortativity, $r$. We see a similar level of dis-assortativity in the maximized and minimized networks, while the empirical network is more assortative and the thresholded empirical network is less assortative. (e) DebtRank, $R$, plotted against the mean local clustering coefficient, $\bar{c}$. We see that there is a tendency toward higher local clustering in the empirical network. The minimized and the thresholded networks show similar average clustering. The average clustering in the maximized networks is slightly higher than in the minimized networks. (f) DebtRank, $R$, plotted against the average weighted nearest neighbor degrees, $\bar{k}_{n n}^{w}$. The smallest values are observed for the minimized network, followed by the maximized networks. The thresholded networks exhibit higher values, the empirical networks substantially higher values. (For interpretation of the references to color in this figure legend, the reader is referred to the web version of this article.) 
We report the average of the ten assortativity coefficients for the four network types: empirical -0.23 , minimized -0.37 , maximized -0.39 , and thresholded -0.47 . We see that the minimized and maximized networks tend to have a similar degree assortativity, whereas the empirical networks are more assortative, and the thresholded network more disassortative.

A similar picture is found for the clustering coefficient, $\bar{c}$. We compute it from the unweighted undirected (symmetrized) adjacency matrix for each of the ten quarters. The clustering coefficient of the network is defined as $\bar{c}=1 / N \sum_{i=1}^{N} C_{i}$, where the local clustering coefficient $C_{i}$ of node $i$ is defined as the number of connected pairs of neighbors of $i$, divided by the number of pairs of neighbors of $i$. Fig. 4(e) plots the clustering coefficients $\bar{c}$ for each quarter against the DebtRank, $R$, of the respective quarter. The average of the clustering coefficients for the ten quarters is 0.86 for the empirical, 0.54 for the minimized, 0.58 for the maximized, and 0.54 for the thresholded network. Again, the average clustering coefficient for the minimized and maximized networks shows a similar behavior. To give a more detailed picture of the clustering behavior, we show the histogram of local clustering coefficients, $C_{i}$, for the different network types pooled for all ten observations in Fig. D.11 (c). Approximately in line with these numbers, in their theoretical model Leduc and Thurner (2017) report average clustering coefficients for 500 networks of about $\bar{c} \sim 0.7$, for networks with usual systemic risk levels, while for networks that are obtained under a systemic risk tax (that systematically reduces systemic risk by incentivizing agents), they find $\bar{c} \sim 0.3$.

Finally, in Fig. 4(f) we study the average-weighted nearest neighbor degree, $\bar{k}_{n n}^{w}=\sum_{i=1}^{N} k_{n n, i}^{w}$, where $k_{n n, i}^{w}$ is defined as $k_{n n, i}^{w}=\left(a_{i}+l_{i}\right)^{-1} \sum_{j=1}^{N}\left(L_{i j}+L_{j i}\right) k_{j}$. The degree of the neighbors of node $i$ are weighted with the size of the mutual exposure between them and are standardized by the sum of is' interbank liabilities and assets. We report the values for the empirical 63, minimized 16, maximized 21, and thresholded 30 networks. For the mean weighted nearest neighbor degree a notable difference between the minimized and maximized networks is observed. However, both types exhibit much smaller values than the empirical networks. The distribution of $k_{n n, i}^{w}$ across all ten observations is shown in Fig. D.11(d).

These findings in the network topology measures clearly indicate that there are substantial differences between the optimized and the empirical networks. Interestingly, the topology measures for minimized and maximized networks are often similar. Not unexpectedly, due to the large number of small exposures in empirical networks, the thresholded networks often appear more similar (based on various network measures) to the optimized ones than to the empirical ones.

\subsection{DebtRank differences and link densities}

We find that sparse networks can exhibit either very low or very high systemic risk. As the ambiguous relationship between link density and systemic risk is one the most frequently discussed relations of network topology and systemic risk (see e.g., Aldasoro et al., 2017; Allen and Gale, 2000; Battiston et al., 2012a; Battiston et al., 2012b; Glasserman and Young, 2016 and references therein), we take a closer look at this issue. Fig. 4 (c) could imply a high variance of DebtRank for small link densities or a highly non-linear relationship. To find out, we conduct a simulation study to investigate the bivariate distribution of the two network characteristics (see Appendix F).

Fig. 5 (a) shows the results for a sample of 200,000 networks depicted in a heat map corresponding to observation Q1. These samples have the same in- and out-strengths as the empirical and optimized networks. The variance is considerably higher in the area of low link density and decreases for higher values. The general relationship appears to be concave with on average highest DebtRanks for intermediate levels of link density. An obvious shortcoming of our sampling method is that the empirical networks lie outside the sample range, indicating that the sample space is not fully explored with the employed algorithm. Fig. F.13(b) shows the same plot for all ten observations with similar patterns across all quarters. The simulation results suggest that the relation between systemic risk and link density is not a simple function-where a given value of link density yields a unique value of systemic risk-but is stochastic, where the mean and variance depend on link density. Clearly, a large part of the variation in systemic risk remains unexplained by link density. A better measure for explaining the large variations in DebtRank is presented next.

Fig. E.12 indicates that DebtRank-weighted assortativity changes during the optimization. In the empirical network most nodes with high DebtRanks are connected. After the optimization, borrowing and lending between high DebtRank nodes is visibly reduced. The DebtRank-weighted assortativity coefficient $r_{\mathrm{DR}, 0.25}$ for the $25 \%$ of banks with highest DebtRank is computed in a similar way to the degree assortativity. The difference is that the weights are changed to $v_{l}=R_{i}$ and $w_{l}=R_{j}$ and only links, $L_{i j}$, for which either $i$ or $j$ belongs to the $25 \%$ of banks with highest DebtRank are taken into account. The relationship is robust to varying the percentage of $25 \%$ between $10 \%$ and $50 \%$.

In Fig. 5(b) the relationship of DebtRank $R$ and assortativity, $r_{\mathrm{DR}, 0.25}$, is shown for the empirical (red squares), minimized (blue triangles) and maximized (green diamonds) networks. The proposed measure clearly separates the three groups from one other. The correlation coefficient for all 30 observations pooled together is $\rho=0.95\left(p\right.$-value $\left.=4 \cdot 10^{-16}\right)$ and suggests that there exists a statistically significant relationship between the two variables ${ }^{9}$. Thus, we find statistical

\footnotetext{
${ }^{9}$ This relationship does not hold within the minimized networks where the correlation is not significantly different from zero. The same holds true also for the maximized group ( $p$-value $=0.057$ ). In the case of the empirical networks the correlation is statistically different from zero with $\rho=0.8$ ( $p$-value $=0.006)$.
} 

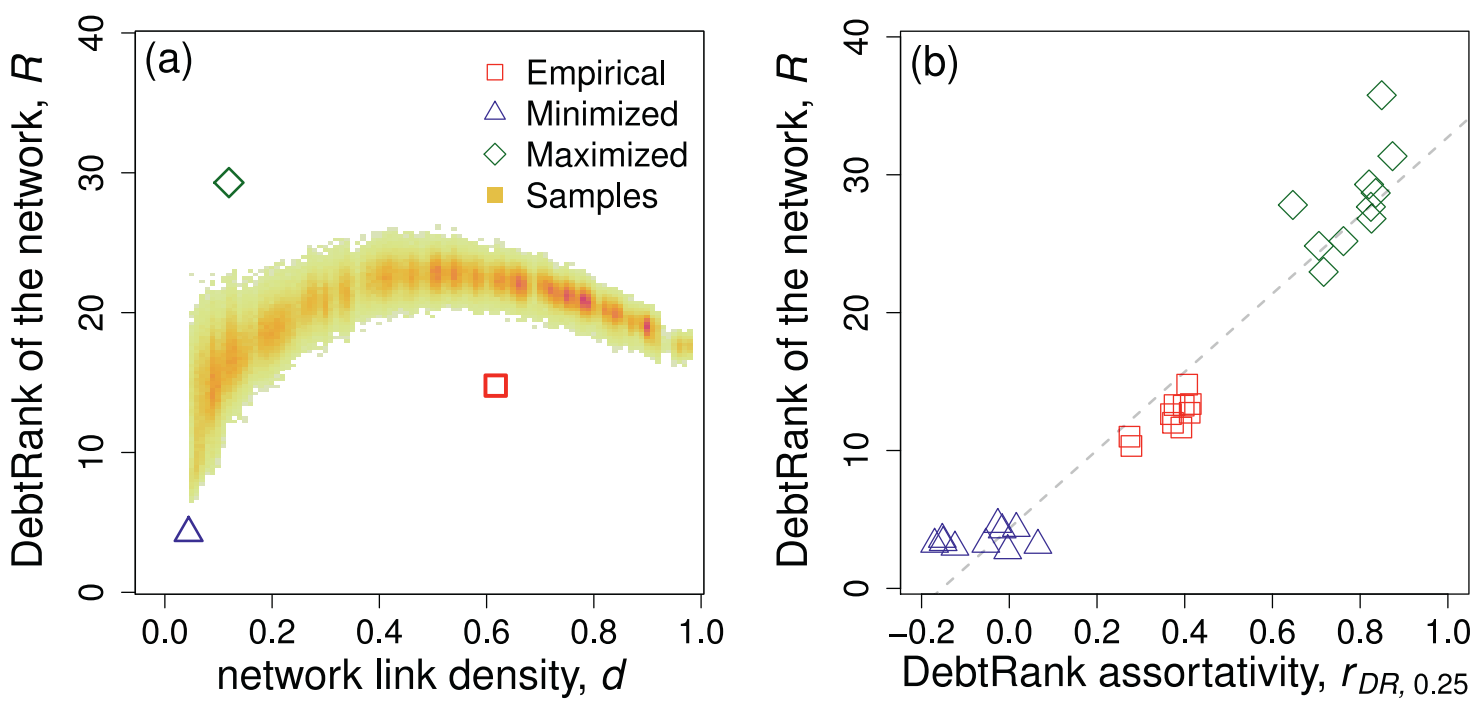

Fig. 5. (a) DebtRank versus link density for the empirical (red squares), minimized (blue triangles), maximized (green diamonds), and randomly sampled networks (orange heat-map; darker coloring indicates that more sampled networks lie in this area). It shows the relationship for the observation in Q1 and 200,000 randomly sampled networks with the same in- and out-strengths. The variance is visibly higher for sparse networks and decreases with link density. Additionally, a concave shape is apparent peaking at density levels around 0.5. The optimized and empirical networks lie outside of the area covered by the sample. (b) Depicts the relationship between DebtRank, $R$, of the empirical (red squares), optimized minimal (blue triangle) and optimized maximal (green diamonds) and DebtRank weighted assortativity coefficient, $r_{\mathrm{DR}, 0.25}$. Clearly, the groups separate in both DebtRank and DebtRank assortativity. There is a clear linear relationship visible if the three groups are pooled together $(\rho=0.95)$. However, within the network types there are considerable differences. There is no significant explanatory power within the group of minimized networks with $\rho=0.25$. The group of maximized networks shows a weakly significant linear relation with $\rho=0.62$ and a $p$-value of 0.06 . The linear relationship is most significant for the group of empirical networks with $\rho=0.8$ and $p$-value $=0.006$.

evidence that DebtRank-weighted assortativity for large banks explains a large part of the variation in DebtRank in the 30 networks.

\section{Discussion of results}

Previous studies discussed the possibility of rearranging financial networks to mitigate financial contagion. We briefly compare their results. Pichler et al. (2020) find that systemic risk (DebtRank) from overlapping portfolio contagion can be reduced by a factor of 2.3 for a data set containing 49 major European banks invested in 36 different sovereign bonds. Poledna and Thurner (2016) show in an agent-based model that the introduction of a network-based systemic risk tax (SRT) reduces systemic risk (DebtRank) by approximately 50\%. In Krause et al. (2019) for the case of an Italian interbank network with 53 banks, their minimization (maximization) procedure can reduce (increase) the networks' shock multiplier from 1.9 to 1.8 (2.25). Poledna et al. (2017) compare the systemic risk reduction from the SRT with a Basel III scenario with GSIB surcharges. They find that the GSIB surcharges would need to be three times higher to achieve a similar level of DebtRank as the SRT does. We find that in our sample existing bank capital (observed 2006-2008) would need to be scaled up by an average factor of 3.3 to achieve the same level of DebtRank as our optimization. In our case the Basel III scenario (increase in bank equity by a factor of $2.125(=8.5 \% / 4.5 \%)$ ) has 1.6 times higher DebtRank than the optimized networks (see Appendix C). Let us briefly mention two studies showing that capital requirements taking the underlying network structure into account can reduce systemic risk significantly. Alter et al. (2015) find that reallocating 12\% of existing capital requirements based on eigenvector centrality can avoid $15 \%$ of total system losses. Cont et al. (2010) show that targeted capital requirements reduce the contagion index of the $5 \%$ most systemic institutions approximately five times more strongly than homogeneous ones (compare Cont et al. 2010, Fig. 17) when the ratio of total equity in the system divided by interbank volume is slightly increased.

We showed that network structure changes significantly during optimization. The minimized (maximized) networks are sparse, with an average link density of about $4.4 \%$ (11\%), in contrast to the empirical networks that show about $56 \%$. Similarly, Krause et al. (2019) find that their minimized and maximized networks are considerably sparser than the original network (average degree decreases from $\bar{k}=14.4$ to $\bar{k}=2$ ). Aldasoro et al. (2017) find for a network of 20 banks (obtained from a theoretical model, where banks lend/borrow in an optimal way w.r.t. their own utility function) a link density of around $7.3 \%$. This suggests that sparse networks can have potentially both a very low and a very high DebtRank. There are two possible explanations at hand. Either a small increase in link density can result in a high increase in systemic risk or the distribution of systemic risk for low density networks varies widely. In Section Appendix F we find evidence for both, 
a high variation in DebtRank for networks with low link densities and a concave relationship between DebtRank and link density. This means that a reduction in connectivity will not necessarily lead to lower levels of systemic risk.

In the literature non-monotonic relations of systemic risk as a function of link density have been observed before by, for example, Nier et al. (2007), Gai and Kapadia (2010), and Glasserman and Young (2016). Another recurring topic in the literature is the trade-off between diversification of risk at the individual bank level and system-wide stability. Usually, higher link densities are associated with more risk-sharing among agents, which in turn can lead to higher overall systemic risk compared to networks with less risk-sharing (low link density), compare, for example, Allen and Gale (2000), Battiston et al. (2012b), Battiston et al. (2012a), or Aldasoro et al. (2017). The fact that systemic risk and link density are related in involved ways implies that the effect of interbank exposure diversification on system-wide stability strongly depends on the exact network structures.

In general we find that simple topology measures such as link density, clustering coefficients, nearest neighbor degree, or degree assortativity are not capable of explaining the difference between the DebtRank of minimized, empirically observed, and maximized networks. However, the DebtRank weighted assortativity coefficient that we introduced here, computed for systemically important banks, clearly separates high, medium, and low DebtRank networks (see Fig. 5 (b)). This is in line with Krause et al. (2019) who find lower shock multipliers for networks being disassortative w.r.t. interbank leverage.

There are obvious issues that should be investigated further in future work. First, there should be an analysis as to whether those networks, which are formed under the SRT regime in Poledna and Thurner (2016), can be further optimized, and if the two methods (optimization and SRT) yield similar network topologies. Second, additional constraints in the optimization could be of economic interest. For example, keeping the diversification of the agents' exposures constant to avoid concentration risk. This could be ensured by controlling desired link densities with (e.g. $L_{1}$-norm) constraints, or by adding a reward term to the objective function for having additional counterparties. By varying such a reward premium it could be studied how optimal networks change when benefits of diversification (and risk sharing) are taken into account. This could shed further light on the relation between density and systemic risk, by, for example, computing upper and lower bounds of DebtRank for given values of link density. Third, it should be investigated whether approaches yielding optimal allocations of capital for given networks and approaches yielding optimal networks for given capital allocations can be combined.

Let us finally mention some limitations of our study. As the data set is anonymized we used banks' leverage ratios as a proxy of credit risk instead of probabilities of default. We assigned the credit riskiness of every bank in a static way before the optimization and assume that the rewiring of links does not change credit riskiness. Another shortcoming is that the maturity of exposures is ignored in the optimization, due to a lack of data. Finally, we considered a single layer of exposures only, even though it is known that systemic risk strongly depends on other exposure layers see, for example, León et al. (2014), Poledna et al. (2015), Molina-Borboa et al. (2015), and Poledna et al. (2018). An interesting extension to this present work would be to understand the effect of multiple exposure layers and their interactions on the minimum of attainable systemic risk. It is conceivable that optimization becomes technically much more challenging for multi-layer networks.

\section{Conclusion and policy implications}

Systemic risk can arise through two channels: synchronization of agents' behavior (e.g., fire sales, or simultaneously stopping interbank lending); and interconnectedness of banks' balance sheets by financial contract networks (e.g. interbank loans, equity crossholdings, or derivative exposures). Here, we quantified how much systemic risk can be mitigated in such financial contract networks by optimally rewiring them with mixed integer linear programming. The restructuring of networks keeps the overall economic conditions of banks unchanged (their capital buffers, total interbank assets and liabilities, and average risk-weighted exposure). The resulting reduction of DebtRank by about 70\%, shows the huge potential for mitigating financial contagion by restructuring financial networks. Existing capital levels would need to be scaled up by a factor of 3.3 to obtain similar levels of systemic risk reduction. These findings emphasize the importance of macro-prudential rules that focus on the structure of financial networks, and demonstrate that simply scaling up capital requirements might not be sufficient to prevent future systemic events. However, this does not mean that capital requirements should be reduced once optimal networks are in place. Capital requirements are needed as buffers against the inherent risks of financial intermediation. To be clear, we do not suggest that real interbank networks should be engineered with the optimization procedure presented. In practice, systemically safe network structures can be achieved by incentivizing transactions that introduce comparably small amounts of systemic risk to the system. Proposals for such incentive schemes were made in Poledna and Thurner (2016) and Leduc and Thurner (2017). The optimization procedure presented here could, however, be helpful in calibrating such incentive schemes. In computer experiments it can be shown how close a given incentive parameter brings the system to the optimally attainable systemic risk levels.

The optimization procedure presented allows us to compare real world networks with networks with minimal and maximal systemic risk. This comparison provides new insights into (macroprudential) regulation. First, we find that simple topological measures, like link density, degree assortativity, or clustering coefficient, fail to explain the large differences in systemic risk across the three network types. Second, simply reducing the interconnectedness of networks (link density) does not necessarily lead to lower levels of systemic risk, as the opposite could also happen. Third, in line with intuition there is evidence that if the most systemically important banks in the network are tightly connected (high DebtRank assortativity) then overall systemic risk in these networks is higher. Fourth, and maybe unsurprisingly, changing the network 
structure seems to address the "too central to fail" problem. In the optimized networks the DebtRank levels of individual banks become "more" proportional to their total interbank liabilities, whereas in empirical networks small banks can have unnecessary high levels of DebtRank. However, in the optimized networks, banks with the largest total liabilities remain systemic ("too big to fail"). Finally, the optimized networks can serve as a benchmark for empirical networks. It can be evaluated how far away an observed network is from the optimum and if over time the system is moving toward the optimum or away from it. In this way the new method can evaluate the effectiveness of implemented policies.

\section{Acknowledgements}

CD acknowledges funding from the WWTF project Stochastic Filtering and Corporate and Sovereign Credit Risk project number MA14-031, PI Rüdiger Frey, and the OeNB anniversary fund project Dynamic measures of systemic risk, project number 17793, PI Birgit Rudloff. ST and CD acknowledge support from the OeNB anniversary fund project Data-driven multi-layer network approaches to quantify the spreading of systemic risk, project number 17795, and ST acknowledges support from FFG project under 857136. AP acknowledges support from Partners for a New Economy and Baillie Gifford.

\section{Appendix A. Details on the MILP}

This section contains the details regarding the structure of the constraint matrices ${ }^{10}$ introduced in Section 3 and visualizes the behavior of the objective function as mentioned in Section 3. Matrix $A_{1}$ contains the constraints corresponding to the reformulation of the $\min ($.$) in the objective function, Eq. (7). Consider the constraints from Eq. (15) to (18)$

$$
\begin{aligned}
y_{2 i-1} & \geq \delta_{2 i} \bar{e}_{i}, \\
y_{2 i-1} & \leq \delta_{2 i-1} \bar{e}_{i}, \\
y_{2 i} & \leq \delta_{2 i} u_{i}, \\
\delta_{2 i-1} & \geq \delta_{2 i},
\end{aligned}
$$

where $u_{i}=\max \left(0, \min \left(\bar{a}_{i}, \bar{l}_{i}\right)-\bar{e}_{i}\right)$. We have to consider that the size of every pair $\left(y_{2 i-1}, y_{2 i}\right)$, representing one value in the liability matrix is constrained by the respective row and column sums of the matrix, and the equity value of the borrowing bank. We recall that the correspondence of the variables is $L, x, y$ is

$$
L_{k l}=L_{\left(i-\left(\left\lceil\frac{i}{n}\right\rceil-1\right) n,\left\lceil\frac{i}{n}\right\rceil\right)}=x_{i}=y_{2 i-1}+y_{2 i}
$$

Let us denote the upper bound for $y_{2 i}$ by $u_{i}=\max \left(0, \min \left(\bar{a}_{i}, \bar{l}_{i}\right)-\bar{e}_{i}\right)$, and recall that the upper bound for $y_{2 i-1}$ is $\bar{e}_{i}$. The first $2 N^{2}$ columns correspond to the $y$ variables. The second $2 N^{2}$ columns correspond to the vector of dummy variables $\delta$, which indicate if the respective $y_{j}$ is positive or zero. For each entry of the matrix $L$ and the corresponding $x_{i}$ there are four

\footnotetext{
10 We provide the $\mathrm{R}$ code for creating the constraint matrices and an example with simulated data on https://csh.ac.at/vis/code/network_optimization/
}

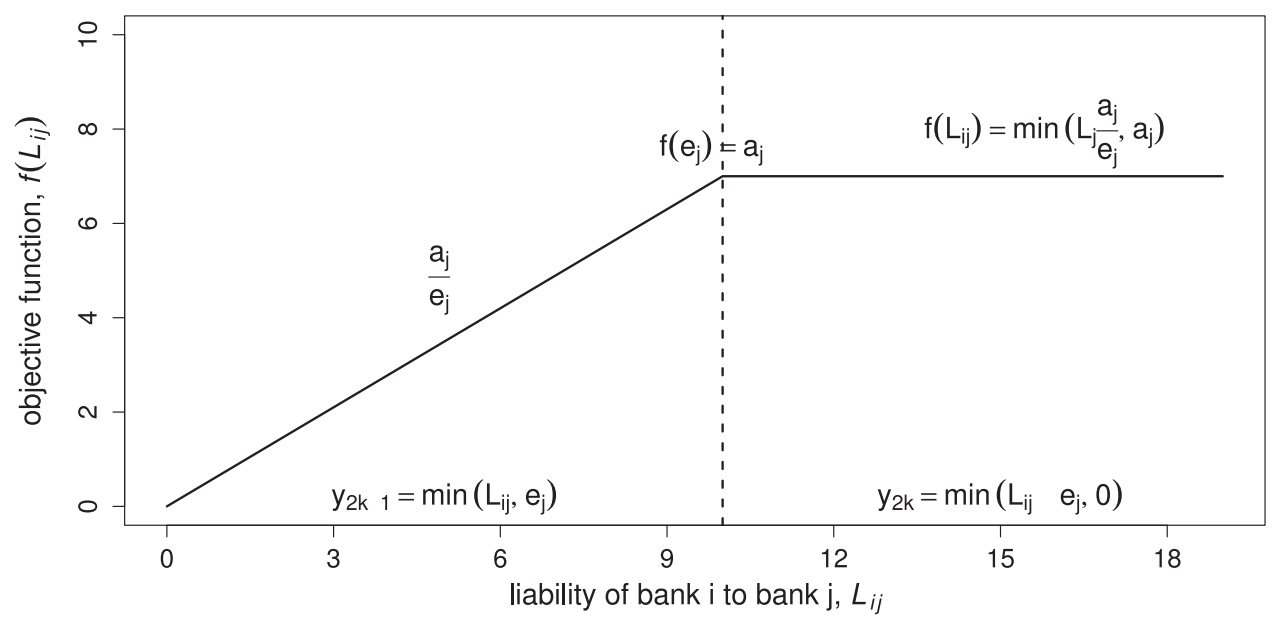

Fig. A.6. Visualization of a single term of the objective function, $f\left(L_{i j}\right)=\min \left(L_{i j} \frac{a_{j}}{e_{j}}, a_{j}\right)$. For the example we set $e_{j}=10, a_{j}=7$. The slope for $L_{i j} \in[0,10)$ is $a_{j} / e_{j}$, and 0 , for $L_{i j}>10$. For $L_{i j}=e_{j}$, we have that $f\left(L_{i j}\right)=a_{j}$, which is the maximal functional value. The two variables $y_{2 k-1}$ and $y_{2 k}$ are defined as $y_{2 k-1}=\min \left(L_{i j}, e_{j}\right)$, and $y_{2 k}=\min \left(L_{i j}-e_{j}, 0\right)$ 
rows enforcing the constraints from Eq. (15) to (18). Note that the diagonal elements of $L$ are forced to zero by specifying the upper bound and lower bound in the optimization software to zero. The structure of $A_{1}$ is schematically depicted as

\begin{tabular}{|c|c|c|c|c|c|c|c|c|c|c|c|c|c|c|c|c|}
\hline & 1 & 2 & & $2 i-1$ & $2 i$ & & $2 N^{2}-1$ & $2 N^{2}$ & $2 N^{2}+1$ & $2 N^{2}+2$ & & $2 N^{2}+2 i-1$ & $2 N^{2}+2 i$ & & $4 N^{2}-1$ & $4 N^{2}$ \\
\hline 1 & 1 & 0 & 0 & 0 & 0 & 0 & 0 & 0 & $-\bar{e}_{1}$ & 0 & 0 & 0 & 0 & 0 & 0 & 0 \\
\hline 2 & -1 & 0 & 0 & 0 & 0 & 0 & 0 & 0 & 0 & $\bar{e}_{1}$ & 0 & 0 & 0 & 0 & 0 & 0 \\
\hline 3 & 0 & 1 & 0 & 0 & 0 & 0 & 0 & 0 & 0 & $-u b_{1}$ & 0 & 0 & 0 & 0 & 0 & 0 \\
\hline 4 & 0 & 0 & 0 & 0 & 0 & 0 & 0 & 0 & -1 & 1 & 0 & 0 & 0 & 0 & 0 & 0 \\
\hline $4 i-3$ & 0 & 0 & 0 & 1 & 0 & 0 & 0 & 0 & 0 & 0 & 0 & $-\bar{e}_{i}$ & 0 & 0 & 0 & 0 \\
\hline $4 i-2$ & 0 & 0 & 0 & -1 & 0 & 0 & 0 & 0 & 0 & 0 & 0 & 0 & $\bar{e}_{i}$ & 0 & 0 & 0 \\
\hline $4 i-1$ & 0 & 0 & 0 & 0 & 1 & 0 & 0 & 0 & 0 & 0 & 0 & 0 & $-u b_{i}$ & 0 & 0 & 0 \\
\hline $4 i$ & 0 & 0 & 0 & 0 & 0 & 0 & 0 & 0 & 0 & 0 & 0 & -1 & 1 & 0 & 0 & 0 \\
\hline $4 N^{2}-3$ & 0 & 0 & 0 & 0 & 0 & 0 & 1 & 0 & 0 & 0 & 0 & 0 & 0 & 0 & $-\bar{e}_{N^{2}}$ & 0 \\
\hline $4 N^{2}-2$ & 0 & 0 & 0 & 0 & 0 & 0 & -1 & 0 & 0 & 0 & 0 & 0 & 0 & 0 & 0 & $\bar{e}_{N^{2}}$ \\
\hline $4 N^{2}-1$ & 0 & 0 & 0 & 0 & 0 & 0 & 0 & 1 & 0 & 0 & 0 & 0 & 0 & 0 & 0 & $-u b_{N^{2}}$ \\
\hline $4 N^{2}$ & 0 & 0 & 0 & 0 & 0 & 0 & 0 & 0 & 0 & 0 & 0 & 0 & 0 & 0 & -1 & 1. \\
\hline
\end{tabular}

The matrix $A_{2}$ is responsible for the column sum constraint $A_{2} z=a$. Note that $A_{2} \in \mathbb{R}_{+}^{N \times 4 N^{2}}$, where the last $2 N^{2}$ columns are zero columns as the binary vector is not in use here. As the diagonal elements of $L$ are set to zero, the correct column sum must be achieved through the other $N-1$ entries in the respective column. As $x=$ $\left(L_{11}, \ldots, L_{N 1}, L_{12} \ldots, L_{N 2}, \ldots, L_{1 N}, \ldots, L_{N N}\right)^{\top}$, the first $N$ entries of $x$ correspond to the first column of $L$, the next $N+1$ to $2 N$ entries to the second column, and so on. This translates again to $y$ and the first $2 N^{2}$ columns of $A_{2}$ follow the diagonal structure below. For a more compact notation ${ }^{11}$ of $A_{2}$, we define the vectors $\iota_{i}$ of length $2 \mathrm{~N}$ as

$$
\begin{aligned}
\iota_{1} & =\left(0_{2}, 1_{2 N-2}\right) \\
\iota_{2} & =\left(1_{2}, 0_{2}, 1_{2 N-4}\right) \\
\iota_{i} & =\left(1_{2 i-2}, 0_{2}, 1_{2 N-2 i}\right) \\
\iota_{n} & =\left(1_{2 N-2}, 0_{2}\right) .
\end{aligned}
$$

Where $0_{2}=(0,0)$ and $1_{i}=(1, \ldots, 1)$ of length $i$. Then, $A_{2}$ has the following structure

$$
A_{2}=\left[\begin{array}{ccccccc}
\iota_{1} & 0 & \ldots & 0 & 0 & 0 & 0_{2 n^{2}} \\
0 & \iota_{2} & 0 & \ldots & 0 & 0 & 0_{2 n^{2}} \\
\vdots & 0 & \ddots & \ddots & & \vdots & 0_{2 N^{2}} \\
0 & \vdots & \ddots & \iota_{i} & 0 & 0 & 0_{2 N^{2}} \\
0 & 0 & & 0 & \ddots & 0 & 0_{2 N^{2}} \\
0 & 0 & \ldots & 0 & 0 & \iota_{N} & 0_{2 N^{2}}
\end{array}\right],
$$

and as the rows of $A_{2}$ are of length $4 N^{2}, 0$ must be of length $2 N$, as $\iota$.

Matrix $A_{3}$ enforces the constraints on row sums and has a similar structure to $A_{2}$. Again the first $2 N^{2}$ entries correspond to the $y$ values, while the latter ones correspond to the binary variables and are zero. Again, we set $0_{2}=(0,0)$ and $1_{2}=$ $(1,1)$, and define a sequence of auxiliary matrices $B_{1}, B_{2} \ldots, B_{N}$ with dimension $N \times 2 N$ as

$$
B_{1}=\left[\begin{array}{cccccc}
0_{2} & 0_{2} & \ldots & 0_{2} & 0_{2} & 0_{2} \\
0_{2} & 1_{2} & 0_{2} & \ldots & 0_{2} & 0_{2} \\
\vdots & 0_{2} & \ddots & \ddots & & \vdots \\
0_{2} & \vdots & \ddots & 1_{2} & 0_{2} & 0_{2} \\
0_{2} & 0_{2} & & 0_{2} & \ddots & 0_{2} \\
0_{2} & 0_{2} & \ldots & 0_{2} & 0_{2} & 1_{2}
\end{array}\right] .
$$

\footnotetext{
${ }^{11}$ In the following we slightly abuse notation and use the subscript for defining the dimension of vectors having a constant value.
} 


$$
\begin{aligned}
B_{2} & =\left[\begin{array}{cccccc}
1_{2} & 0_{2} & \ldots & 0_{2} & 0_{2} & 0_{2} \\
0_{2} & 0_{2} & 0_{2} & \ldots & 0_{2} & 0_{2} \\
\vdots & 0_{2} & 1_{2} & \ddots & & \vdots \\
0_{2} & \vdots & \ddots & \ddots & 0_{2} & 0_{2} \\
0_{2} & 0_{2} & & 0_{2} & 1_{2} & 0_{2} \\
0_{2} & 0_{2} & \ldots & 0_{2} & 0_{2} & 1_{2}
\end{array}\right] . \\
B_{i} & =\left[\begin{array}{ccccccc}
1_{2} & 0_{2} & \ldots & 0_{2} & 0_{2} & 0_{2} & 0_{2} \\
0_{2} & \ddots & 0_{2} & \ldots & 0_{2} & 0_{2} & 0_{2} \\
\vdots & 0_{2} & 1_{2} & \ddots & & \vdots & 0_{2} \\
0_{2} & \vdots & \ddots & 0_{2} & 0_{2} & 0_{2} & 0_{2} \\
0_{2} & 0_{2} & & 0_{2} & 1_{2} & \ddots & 0_{2} \\
0_{2} & 0_{2} & \ldots & 0_{2} & \ddots & \ddots & 0_{2} \\
0_{2} & 0_{2} & 0_{2} & 0_{2} & 0_{2} & 0_{2} & 1_{2}
\end{array}\right] .
\end{aligned}
$$

Then $A_{3}$ can be defined as block matrix

$$
A_{3}=\left[\begin{array}{lllllll}
B_{1} & B_{2} & \ldots & B_{i} & \ldots & B_{N} & 0_{2 N^{2} \times 2 N^{2}}
\end{array}\right]
$$

Matrix $A_{4}$ is responsible for keeping the credit risk-weighted exposure to other banks constant for each interbank loan portfolio. Recall that the credit riskiness of bank $i$ is $\kappa_{i}$, and the credit risk weighted exposure of the empirically observed matrix is $r=L^{\top} \kappa$. Then, $A_{4}$ is a $\kappa$-weighted version of $A_{2}$. Let

$$
\begin{gathered}
v_{1}=\iota_{1} \cdot \kappa \\
v_{2}=\iota_{2} \cdot \kappa \\
\vdots \vdots \quad \vdots \\
v_{N}=\iota_{N} \cdot \kappa,
\end{gathered}
$$

where - denotes the pointwise multiplication of two vectors. Then $A_{4}$ can be defined as

$$
A_{4}=\left[\begin{array}{ccccccc}
v_{1} & 0 & \ldots & 0 & 0 & 0 & 0_{2 N^{2}} \\
0 & \nu_{2} & 0 & \ldots & 0 & 0 & 0_{2 N^{2}} \\
\vdots & 0 & \ddots & \ddots & & \vdots & 0_{2 N^{2}} \\
0 & \vdots & \ddots & v_{i} & 0 & 0 & 0_{2 N^{2}} \\
0 & 0 & & 0 & \ddots & 0 & 0_{2 N^{2}} \\
0 & 0 & \ldots & 0 & 0 & v_{n} & 0_{2 N^{2}}
\end{array}\right] .
$$

This completes the set of constraints for the MILP.

\section{Appendix B. Robustness of the optimization procedure}

This section analyzes the robustness of the network optimization by perturbing the empirical network. This is important for two reasons. First, it is desirable for the optimization to reduce the DebtRank of similar networks by a similar factor to provide reliable benchmarks for empirical networks. Second, data on interbank loans often come with uncertainties. For example, there could be reporting errors or thresholds in reporting standards such that only large loans get reported or when data is not available in real time.

The starting point for the robustness check is the empirically observed network, $L$, and its optimized counterpart, $L^{*}$. First, we simulate $k$ perturbed versions $P_{1}, \ldots, P_{k}$ of the empirically observed matrix $L$. Second, we perform the minimization procedure as in Section 4.1 for the networks $P_{1}, \ldots, P_{k}$ and receive the optimized networks $P_{1}^{*}, \ldots, P_{k}^{*}$. Then, for each of those networks we calculate the DebtRank and see how much they differ from the DebtRank of the benchmark networks $L$ and $L^{*}$. We consider two different perturbation scenarios.

In the first scenario only already existing links (i.e., where $L_{i j}>0$ ) are perturbed. We add to each link $L_{i j}$ a normally distributed noise term with zero mean and a standard deviation which is proportional to the link weight, $L_{i j}$, that is, we set

$$
P_{i j}=\max \left[0, L_{i j}+N\left(0, \sigma L_{i j}\right)\right] .
$$



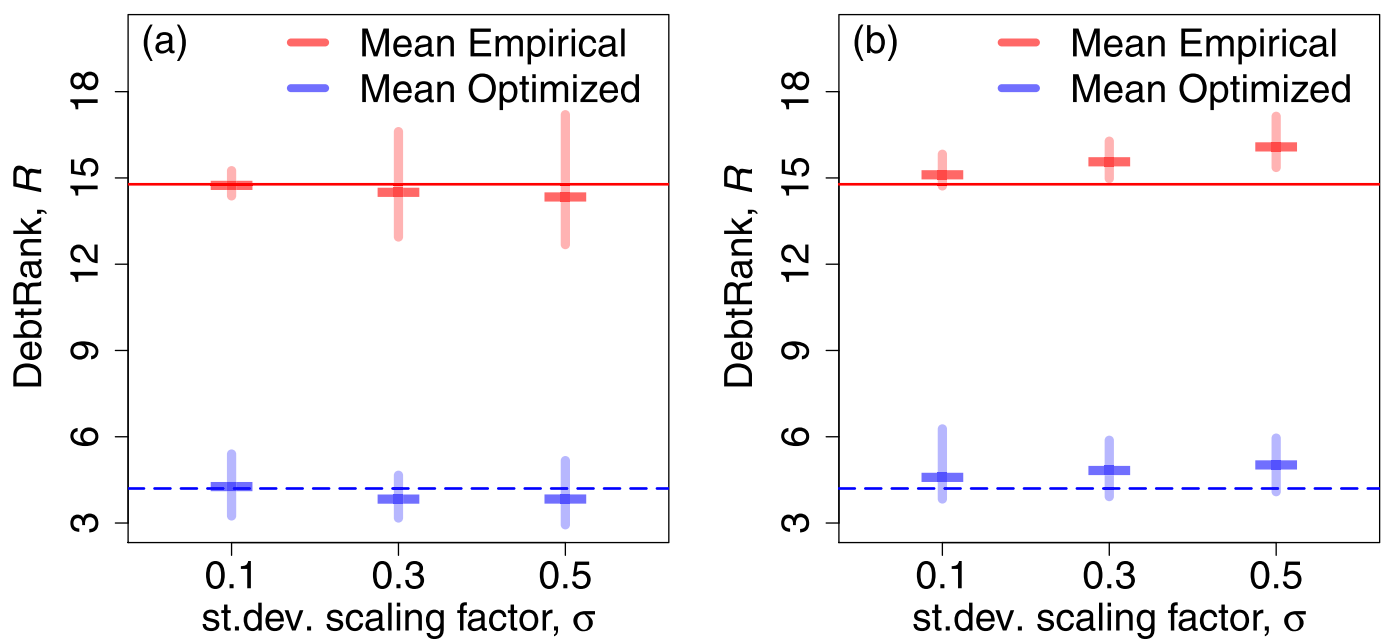

Fig. B.7. Robustness of the optimization procedure with respect to link perturbations for observation Q1. The red solid and the blue dashed horizontal lines correspond to the DebtRank of observation Q1 and of the optimized network corresponding to Q1, respectively. The red and blue bars correspond to the average DebtRank of 100 perturbed networks and the corresponding 100 optimized networks. Vertical lines are the corresponding error bars spanned by the minimum and maximum DebtRank of the respective perturbation sample. We see that across all six perturbation samples the optimization reduces systemic risk significantly. (a) Every already existing link in the network Q1 is perturbed by normally distributed noise with standard deviation $\sigma$. $L_{i j}$. We see that the network optimization is robust and on average reduces DebtRank by a factor of 3.7. (b) Every potential link $L_{i j}$ in the network is perturbed with a probability of $20 \%$ by noise with standard deviation $\sigma \cdot 0.5\left(\bar{s}_{i}^{\text {out }}+\bar{s}_{j}^{\text {in }}\right)$. Again, DebtRank is significantly reduced by the optimization procedure on average by a factor of 3.2. ( (For interpretation of the references to color in this figure legend, the reader is referred to the web version of this article.)

We consider three different levels of noise by setting the standard deviation scaling factor $\sigma$ to $0.1,0.3$, and 0.5 . Note that a value of $\sigma=0.5$ represents very high uncertainty about the actual exposures, which might not be the case in practice. In the second scenario, every possible link can be perturbed, but only with a certain probability, $p$. With probability, $p$, we add to each possible link, $L_{i j}$, where $(i \neq j)$ a normally distributed noise term with zero mean and standard deviation proportional to the sum of the mean out-strength of node $i$ and the mean in-strength of node $j$, that is, we set

$$
P_{i j}=\max \left[0, L_{i j}+B(p) N\left(0, \sigma 0.5\left(\bar{s}_{i}^{\text {out }}+\bar{s}_{j}^{\text {in }}\right)\right],\right.
$$

where $B(p)$ is a Bernoulli distributed random variable, $\bar{s}_{i}^{\text {out }}=(1 / n) \sum_{j=1}^{n} L i j$ is the mean out strength of node $i$, and $\bar{s}_{j}^{\text {in }}=$ $(1 / n) \sum_{i=1}^{n} L_{i j}$ is the mean in strength of node $j$. For our test we set $p=0.2$ and again use three different values for the standard deviation, $\sigma$ to $0.1,0.3$, and 0.5 . Note that in this perturbation scenario new links are created and therefore a high value of $p$ would change the network unrealistically strongly. The expected increase in link density, $d$, is simply $(1-d) p / 2$. For observation Q1 we expect the link density to increase from 62\% to 65.8\%, which amounts to roughly 180 new links. Note that this also increases the interbank volume slightly by about $2 \%$.

The results for observation Q1 are visualized in Fig. B.7, with $k=100$ for each of the six different perturbations. The red horizontal line corresponds to the DebtRank of the empirical observation Q1, and the blue dashed horizontal line to the DebtRank of the minimized network (compare Fig. 2). The red bars represent the mean DebtRank of the 100 perturbed networks $P_{1}, \ldots, P_{100}$, and the blue bars show the mean DebtRank of the matrices $P_{1}^{*}, \ldots, P_{1}^{*} 00$. The vertical transparent lines indicate the smallest and largest DebtRank value of each sample. Overall, the optimization behaves very robustly, even for the large standard deviations.

Fig. B.7 (a) shows the results of perturbation scenario one, where only existing links are perturbed. The figure suggests that the optimization is robust to this type of perturbation. The optimization reduces the DebtRank of the 300 perturbed networks by an average factor of 3.7, supporting the findings from Section 4.1. For all three parameterizations of $\sigma$ the mean DebtRank of the optimized perturbed networks (4.3, 3.8, 3.8, blue bars) are close to the DebtRank of the optimized unperturbed network $L^{*}$ (4.2, blue dashed horizontal line). The standard deviations of the three empirical perturbed samples are $0.18,0.65$, and 0.81 , respectively, compared to $0.53,0.35$, and 0.41 for the respective minimized samples. Fig. B.7(b) shows the results of perturbation scenario two, where all potential links can be perturbed with a probability of $20 \%$. The average DebtRank reduction factor is 3.2. The mean values are again close to the original value. The increase in the means with $\sigma$ stems from a small average increase in the interbank volume from this perturbation scenario. If $L_{i j}=0$, a negative perturbation will have no effect, but a positive perturbation increases the interbank volume. The standard deviations of the three empirical perturbed samples are $0.22,0.27$, and 0.36 , respectively, in comparison to $0.41,0.41$ and 0.44 for the respective minimized samples. We mention that the 600 optimization problems were solved under a time constraint of 5 minutes to foster computational feasibility. The sample size with 100 observations per perturbation is rather small, but across the 600 samples the procedure is satisfyingly robust. 

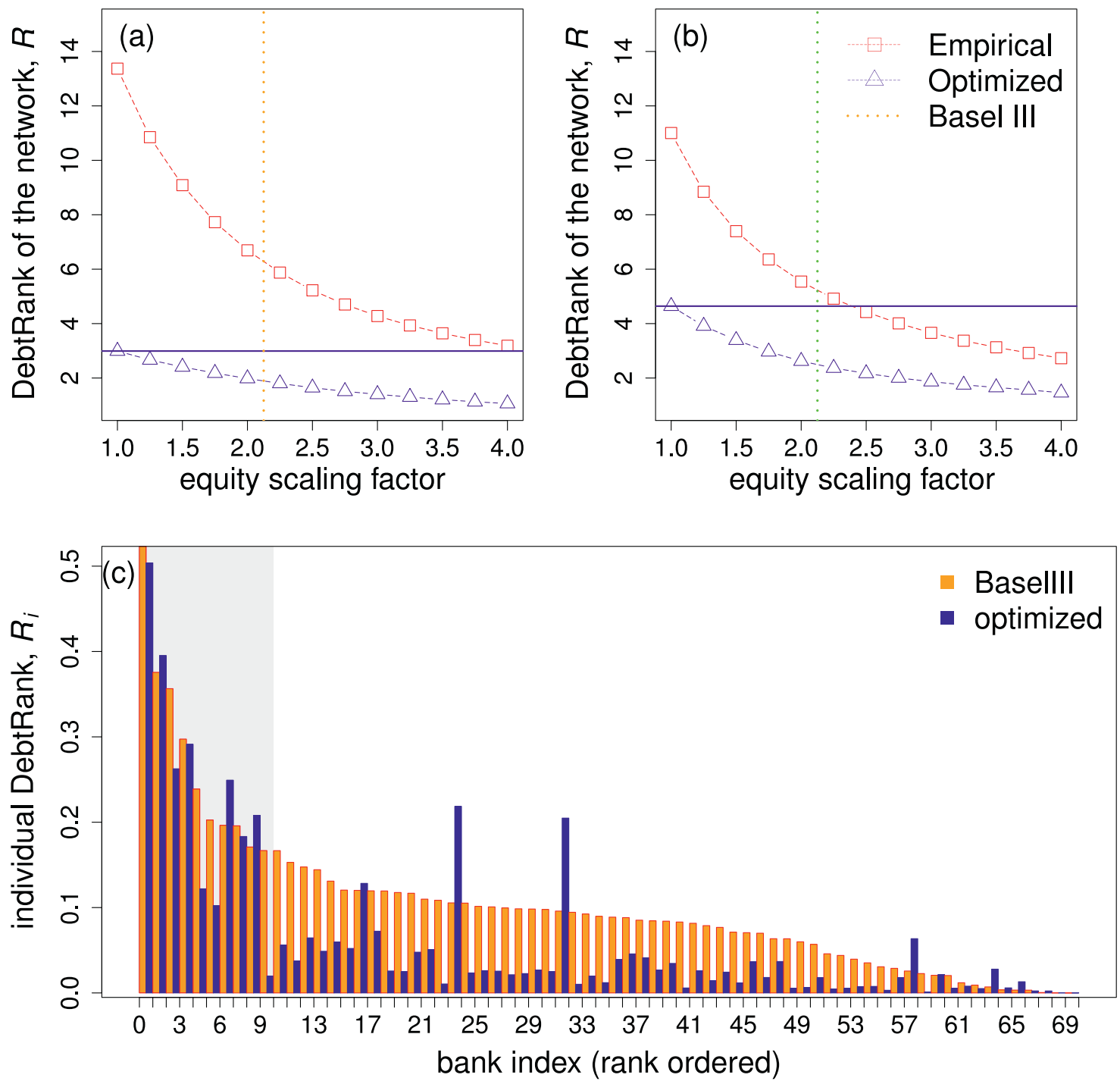

Fig. C.8. Comparison of the effect of capital increases on the DebtRank of empirical and optimized networks for (a) Q7 and (b) Q10. On the $x$-axes the equity scaling factor is shown, and on the $y$-axes the DebtRank is computed for the empirical network (red squares) and optimized network (blue triangles) with the scaled equity vector. The blue horizontal line indicates the DebtRank of the optimized network with the original equity vector (i.e., a scaling factor of 1). The orange vertical dotted line indicates the equity scaling factor 2.125 , corresponding to the increase in Tier 1 capital from $4 \%$ to $8.5 \%$ under Basel III. We calculate the equity scaling factors of 2.38 (Q7) and 4.26 (Q10) as break-even amounts of capital, which reduce the DebtRank of the empirical network to the level of the optimized network (intersection of red dashed line with blue horizontal line). (c) Comparison of the single bank DebtRank $R_{i}$ of the optimized network and the empirical network with equity vector scaled by 2.125 (Basel III) for observation Q1. We see that for certain banks DebtRank $R_{i}$ is higher in the optimized network, but in general the optimization is more effective. DebtRank under the Basel III scenario is 7.13 and 4.25 for the optimized case. (For interpretation of the references to color in this figure legend, the reader is referred to the web version of this article.)

\section{Appendix C. Network optimization versus equity increase}

This section compares the effectiveness of the presented network optimization with increases in bank capital so as to reduce DebtRank. To do this, we perform the following calculations. We increase the equity vector by multiplying it by scaling factors of between one and four in steps corresponding to $25 \%$ increases in equity. Here, we take the approach of increasing the equity of each bank by the same scaling factor. We then calculate the DebtRank for the empirical and the optimized networks with the increased equity vectors. Thus, we observe DebtRank as a function of equity scaling factors as depicted in Fig. C.8. Consequently, we can observe the break-even equity scaling factor, which is required to bring the DebtRank of the empirical network to the level of the optimized network. The smallest and largest break-even factor is realized for observations Q7 and Q10, respectively, which are depicted in Fig. C.8(a) and (b). The required factor for Q7 is 2.38 and for Q10 is 4.26 . The median and mean equity scaling factors are 3.42 and 3.32 . Thus, on average, $232 \%$ of additional bank capital needs to be provided to reduce DebtRank to the level of the optimized networks. 
Fig. C.8 (c) shows the single bank DebtRanks, $R_{i}$, for the optimized case in blue and the empirical network with the equity vector scaled by 2.125 in orange. In most cases the optimization still reduces DebtRank more strongly, but not in all cases. The optimization performs better for the banks with smaller DebtRank. In general DebtRank is lower for the optimized network with 4.25, whereas DebtRank for the Basel III case is 7.13.

\section{Appendix D. Network visualization and topology measures}

This section contains the details of the network visualization and provides additional figures on the effects of the optimization on the network structure.

Fig. D.9(a) shows a visualization of the empirically observed network of the 70 largest Austrian banks for quarter Q1, as does Fig. 4(a) in the main text. The link density is around 62\%. (b) shows the same network, but thresholded such that only the largest links accounting for $90 \%$ of interbank market volumes are used. This reduced network has a link density of about 9.4\%. Fig. D.9(c) shows the minimized network for the same quarter Q1, which has a link density of $4.4 \%$. The respective maximized network is visualized in Fig. D.9(d). It has an link density of around 12\%. In all four panels node size corresponds to the equity of the node, and the link width corresponds to the size of the respective liability, $L_{i j}$. As link- and equity values differ strongly in size, we employed transformations of the quantities to provide a more readable presentation of the networks. Values are first compressed by taking the square root and then standardized by the maximal edge size, $L_{i j}^{\max }=\max \left\{L_{i j}^{0.5} \mid \forall i j \in\{1, \ldots, n\}^{2}\right\}$, and maximal equity size, $E_{i}^{\max }=\max \left\{E_{i}^{0.5} \mid \forall i \in\{1, \ldots, n\}\right\}$, to receive values between zero and one. We then multiply these values by a factor of 15 . Dark red and dark blue nodes indicate high and low DebtRank $R_{i}$,
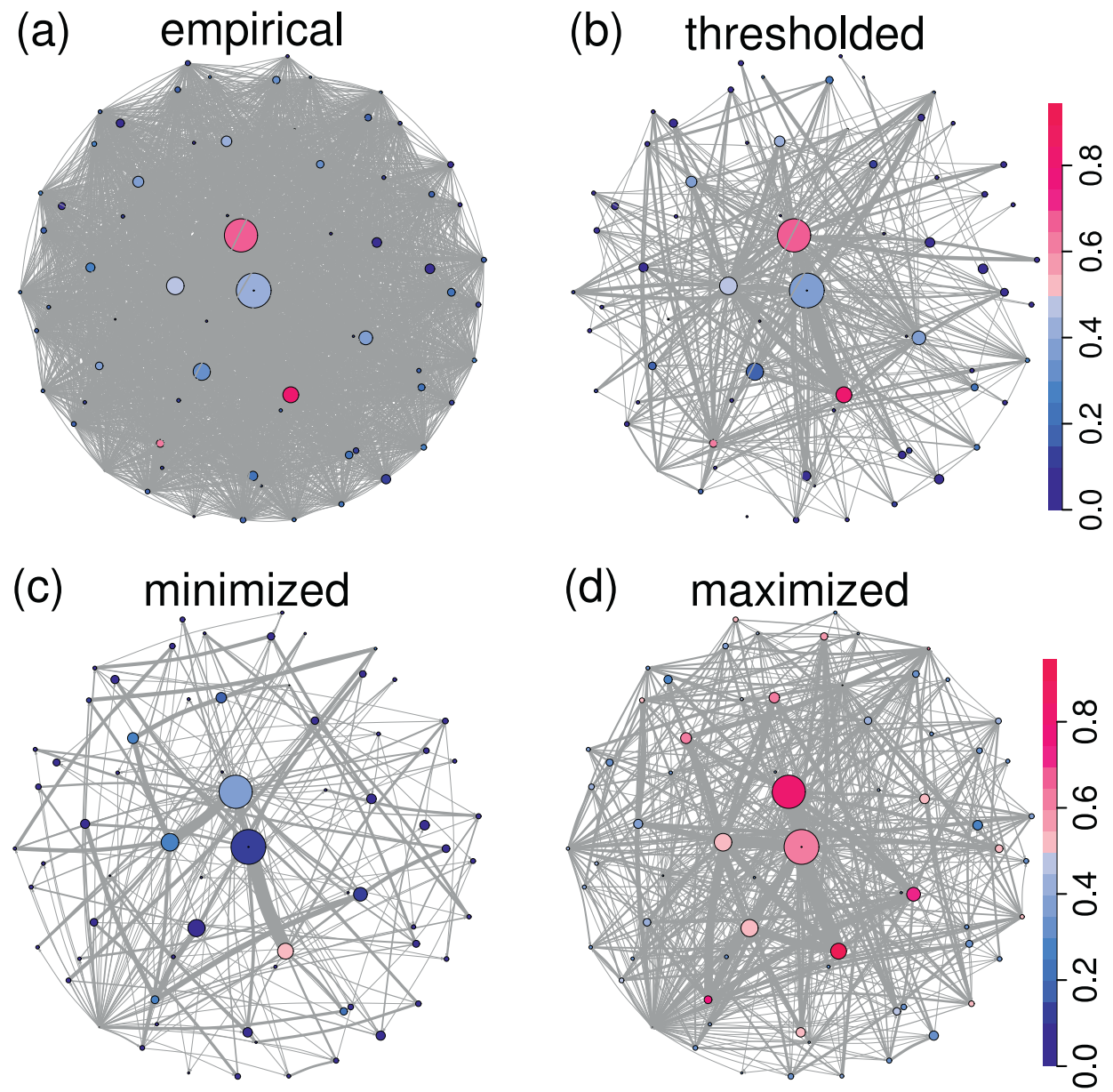

Fig. D.9. Comparison of the empirical asset-liability network, $L$, as observed in Q1 (a), with a thresholded network, where the 454 largest links are shown that cover $90 \%$ of the interbank market volume. The link density decreases from around $62 \%$ in (a) to $9.4 \%$ in (b), which is visibly sparser. (c) Minimized network (same as in Fig. 4(b)). (d) Maximized network in the same quarter. The color represents a high DebtRank (red), low systemic risk is blue. (For interpretation of the references to color in this figure legend, the reader is referred to the web version of this article.) 


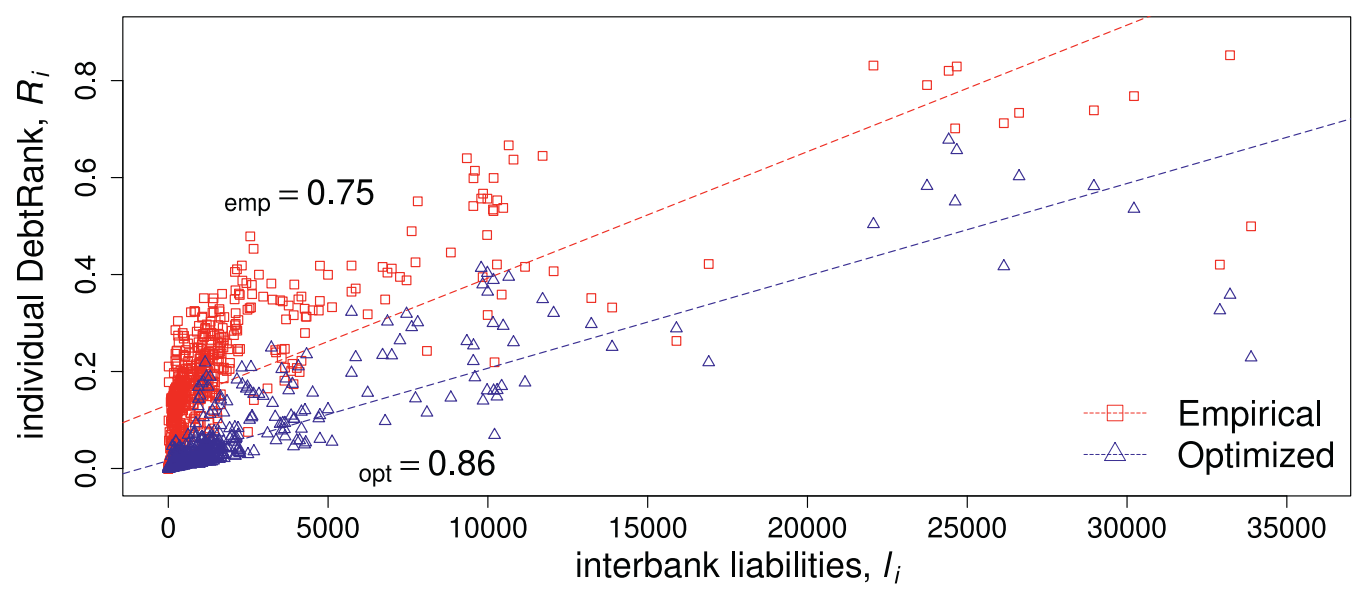

Fig. D.10. Comparison of individual bank DebtRanks, $R_{i}$, with interbank liabilities $l_{i}$ for the empirical (red squares) and the minimized (blue triangles) networks for all ten quarters. It is clear that the optimization strengthens the relationship between banks' interbank liabilities $l_{i}$ and their DebtRank $R_{i}$. In saver networks systemic risk is more proportional to bank size than in the riskier empirical ones. The optimization shows that smaller banks can be rendered systemically negligible by changing the network topology.
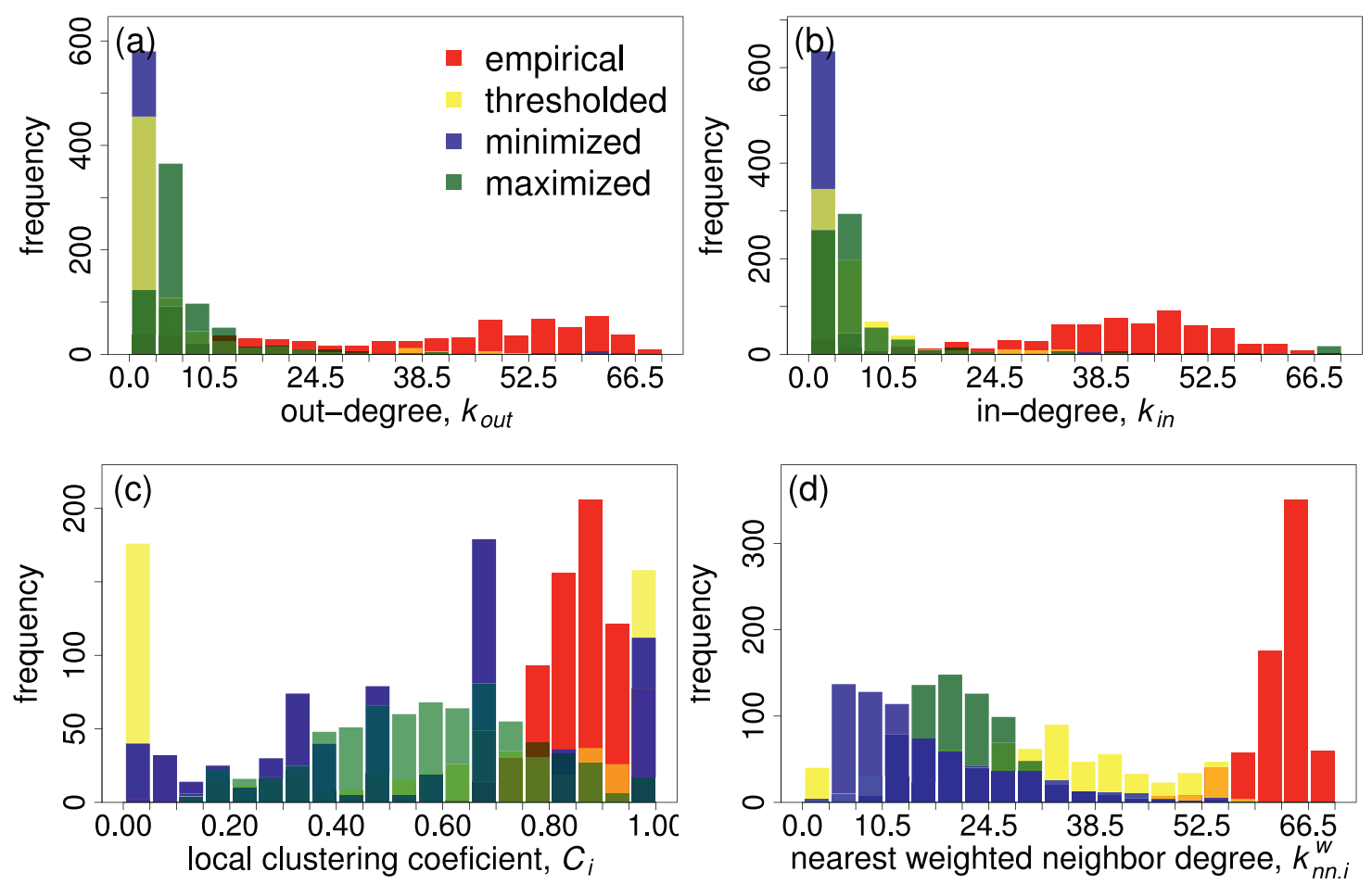

Fig. D.11. (a) shows the distribution of the out-degrees, $k_{\text {out }}$, for the four different network types, empirical (red), minimized (blue), maximized (green), thresholded (yellow). Data is pooled for the ten quarters. (b) in-degree distribution of $k_{\text {in }}$ for the same networks (c) Distribution of the local clustering coefficient, $C_{i}$. There are obvious differences. (d) Distribution of the nearest weighted neighbor degree, $k_{n n i,}^{w}$. (For interpretation of the references to color in this figure legend, the reader is referred to the web version of this article.)

respectively; lighter tones show medium- sized values. The network visualizations were produced with the igraph $\mathrm{R}$ package Csardi and Nepusz (2006).

Fig. D.10 plots the individual bank DebtRanks $R_{i}$ against the banks' interbank liabilities $l_{i}$ for all quarters. In general DebtRanks $R_{i}$ are higher for banks with large interbank liabilities $l_{i}$. It is clearly visible that in the optimized networks, bank DebtRanks are more proportional to the banks' interbank liabilities than in the original network. This is also supported by the higher correlation of 0.86 for the optimized network than for the empirical network where the correlation is 0.75 . For 
the empirical network the relationship looks non-linear and small banks "punch high above their weight." The optimization resolves this issue and renders small banks systemically negligible.

The following Fig. D.11 provides a more detailed perspective on the local network properties such as the in- and outdegree distributions, the clustering behavior, and the weighted nearest neighbor degree for the four different network types. The measures are pooled over all ten observations. Fig. D.11(a) shows the distribution of the out-degrees $k_{\text {out }}$ for the four different network types across all ten observations; empirical (red), minimized (blue), maximized (green), thresholded (yellow). (b) shows the distribution of the in-degrees $k_{\text {in }}$ for the four different network types across all ten observations. In both cases, degrees of the minimized networks are severely peaked in the range of 0 and 3.5. Only at the value of 70 there is a small mass for the out-degree. The maximized networks peak between 3.5 and 7 and are right-skewed. There is small mass at 70 for the in-degree. The empirical network degrees have a wide distribution, with most mass at around 55 for the out-degree and 45 for the in-degree. The thresholded network degrees are peaked at between 0 and 3.5 and decay fast. Fig. D.11(c) shows the distribution of the local clustering coefficient $C_{i}$ for the four different network types. There are obvious differences. The empirical clustering coefficient distribution has the bulk of its mass at values around 0.85 , whereas the thresholded network is bi-modal and has its mass equally divided between zero and one. The distribution for the minimized networks has its mass distributed across the whole spectrum, with peaks at 0.7 and 1 , while the remainder of the mass is allocated at smaller values. The distribution of the maximized networks is rather flat and distributed between 0.2 and 0.8 with a peak at around 0.55. (d) shows the distribution of the weighted nearest neighbor degree $k_{n n . i}^{w}$ for the same networks. Again, there are obvious differences. The $k_{n n, i}^{w}$ for the empirical networks are centered at around 65 , whereas the thresholded network values are distributed between 0 and 60, with a slight peak at around 30 . The values of the minimized network are right-skewed with a peak at around 7. The values for the maximized networks range between 10 and 50 , with a peak at around 20 .

\section{Appendix E. How single links change during the optimization}

The network measures presented in Fig. 4 are shown at the level of the whole network, and Fig. D.11 focuses on the distribution of those measures at the node level. However, it is also of great interest to see how single links change during the optimization. As the optimized networks are considerably sparser, many links that were present originally are expected to disappear, with the creation of potential new links where no link was present before. The probability of a link which is present in the empirical network still being present after the optimization is on average $5 \%$ across the ten observations. The probability of a link existing in the empirical network given, it was created by the optimization, is $62 \%$ on average across the ten observations. The details for the single quarters are presented in Table E.1.

Table E.1 shows statistics of links in the empirical and optimized network across the ten observations. The link densities for the empirical and optimized network and the respective number of links present in the networks is shown in rows one to four. The fifth row shows the number of links present before and after the optimization. From this we can calculate two probabilities, which tell us how likely it is that an initially present link survives after the optimization and how likely it is that the links created by the optimization were already present in the empirical network. The link survival probability is calculated as the number of links that are present in both networks divided by the number of links in the empirical network. The link recreation probability is calculated as the number of overlapping links divided by the number of links in the optimized network. Thus, it gives the probability of a link being recreated by the optimization at a spot where there is a link in the empirical network.

An obvious consequence of the optimized networks being sparser than the empirical ones and the amounts lent and borrowed by the banks (row- and column-sum constraints) staying constant is that the average link weight must increase. The mean link weights are 46.57 and 593.31 for the empirical and optimized network, and the difference in median weight is even starker where we observe 2.69 and 138.46, respectively. Further details about the link weight summary statistics are given in Table E.2.

Table E.2 shows the summary statistics for link weights larger than zero pooled across all ten observations for the empirical, minimized, maximized, and thresholded networks. There are substantial differences. The mean link weights multiplied by the link density must stay constant for the empirical, minimized, and maximized networks. Thus, the minimized network

Table E.1

Statistics of the links in the optimized and empirical networks for all ten observations.

\begin{tabular}{llllllllllll}
\hline Observations & Q1 & Q2 & Q3 & Q4 & Q5 & Q6 & Q7 & Q8 & Q9 & Q10 & Mean \\
\hline density empirical & 0.62 & 0.61 & 0.62 & 0.56 & 0.59 & 0.56 & 0.57 & 0.56 & 0.45 & 0.48 & 0.56 \\
density optimized & 0.04 & 0.04 & 0.04 & 0.04 & 0.04 & 0.04 & 0.04 & 0.05 & 0.04 & 0.04 & 0.04 \\
no. emp. links & 2980 & 2935 & 2974 & 2689 & 2827 & 2699 & 2766 & 2691 & 2196 & 2338 & 2709 \\
no. opt. links & 214 & 211 & 213 & 210 & 209 & 207 & 217 & 221 & 210 & 215 & 212 \\
no. overlap. links & 149 & 139 & 144 & 130 & 131 & 118 & 141 & 142 & 110 & 112 & 131 \\
survival prob. & 0.05 & 0.05 & 0.05 & 0.05 & 0.05 & 0.04 & 0.05 & 0.05 & 0.05 & 0.05 & 0.05 \\
recreation prob. & 0.70 & 0.66 & 0.68 & 0.62 & 0.63 & 0.57 & 0.65 & 0.64 & 0.52 & 0.52 & 0.62 \\
\hline
\end{tabular}


Table E.2

Summary statistics of link weights, which are larger than zero pooled across all ten observations for the empirical, minimized, maximized, and thresholded networks.

\begin{tabular}{lllll}
\hline & Empirical & Minimized & Maximized & Thresholded \\
\hline Min. & 0.04 & 0.00 & 0.00 & 22.45 \\
1st Qu. & 0.60 & 2.66 & 6.01 & 49.58 \\
Median & 2.70 & 138.47 & 51.23 & 108.38 \\
Mean & 46.58 & 593.31 & 237.26 & 301.42 \\
3rd Qu. & 12.76 & 531.19 & 175.17 & 242.58 \\
Max. & 30831.21 & 32903.60 & 22368.02 & 30831.21 \\
\hline
\end{tabular}

has the largest mean, as it is the sparsest network. The median does not fulfil this proportionality condition. We see that median of the minimized network is disproportionately larger for the minimized network than the empirical network.

An interesting aspect is how the relation of the link weight $L_{i j}$ and the DebtRank of the associated outgoing node $R_{i}$ and incoming node $R_{j}$ changes. This is visualized in Fig. E.12 where log link weights $\log _{10}\left(L_{i j}\right)$ are plotted against DebtRank of the outgoing node $R_{i}(\mathrm{a})$ and against DebtRank of the incoming node $R_{j}(\mathrm{~b})$ for the links of observation Q1 before and after the optimization. As outlined earlier, link weights must increase on average for sparser networks with equal node strengths. Due to this fact - and because DebtRank is reduced by the optimization - a shift from many small links between relatively high DebtRank nodes to fewer and larger links associated to relatively low DebtRank nodes is expected. An arrow is drawn between an empirical link (red square) in direction of an optimized link (blue triangle), if this link is present in both networks. Thus, the arrows allow us to see the shift of the 149 overlapping links in this plane. Blue triangles with no arrow pointing at them are newly created links, whereas red triangles with no outgoing arrow are empirical links, which are removed by the optimization.

In (a) the large majority of links from the optimized network (blue triangles) are in the top left quadrant characterized by high link weight and low systemic risk. Most arrows point to the top left with a few exceptions, where link weights stay approximately the same, but DebtRank is lower, or where weight is even reduced. Interestingly, even some of the largest links from the empirical network are still increasing in size. Fig. E.12(b) shows the same general pattern with the difference that there is also a smaller blue cluster visible in the center $\left(0.3 \leq R_{j} \leq 0.4 ; 10^{1} \leq \log _{10}\left(L_{i j} \leq 10^{3}\right) \approx 10^{2}\right)$ where arrows are either pointing from the right above or right below. This indicates that in the optimized network high DebtRank banks have more large incoming links than outgoing links, whereas in the empirical network the high DebtRank banks also have many large outgoing links. Thus, before the optimization the default of the banks with high DebtRank can affect many counterparties and after optimization only very few. This could be evidence that the largest interbank market players are originally relatively strong spreaders of financial losses among each other, whereas after the optimization they turn rather into relative sinks of financial losses.

Fig. E.12 suggests that the assortativity of large DebtRank banks changes in the optimization. This is investigated in more detail in Fig. 5 (a). In the empirical network most nodes with high DebtRanks are connected (red triangles in top right quadrant). After the optimization borrowing and lending between high DebtRank nodes is visibly reduced and the high DebtRank nodes have mainly incoming links from low DebtRank nodes. This pattern can be quantified in terms of a DebtRank weighted assortativity coefficient $r_{D R, 0.25}$ for the bank of the upper DebtRank quartile. It is computed similarly to the degree assortativity with the difference that the weights are now $v_{l}=R_{i}$ and $w_{l}=R_{j}$. Only the links $L_{i j}$ for which either $i$ or $j$ belongs to the $25 \%$ of the banks with highest DebtRank are taken into account. For the observation Q1 in (a) we report $r_{D R .0 .25}=0.41$ for the empirical network and $r_{D R .0 .25}=-0.02$ for the optimized network, which supports our visual conjecture. In (b) the relationship of DebtRank $R$ and assortativity $r_{D R, 0.25}$ is presented for all observations of the empirical (red squares), minimized (blue triangles), and maximized (green diamonds) networks. The first observation is that the pattern revealed by (a) consistently holds throughout all ten observations. Secondly, the maximized networks also have a steadily higher assortativity coefficient $r_{D R, 0.25}$. This suggests that this type of assortativity considering only the high DebtRank nodes could be one of the key drivers of DebtRank. The correlation coefficient for all 30 observations pooled together is $\rho=0.95$ ( $p$-value $=4 \cdot 10^{-16}$ ) and suggests that there is a relationship between the two variables. However, when looking into the intragroup correlations, it is obvious that this relationship clearly does not hold within the minimized networks where the correlation is not significantly different from zero. The same holds true also for the maximized group at the 5\% confidence level ( $p$-value $=0.057$ ). In the case of the empirical networks the correlation is statistically different from zero with $\rho=0.8$ ( $p$-value $=0.006$ ). Thus, while there seems to be statistical evidence that the DebtRank weighted assortativity for large banks has some explanatory power to distinguish between the three groups, the effect is less pronounced within these groups. Another explanation would be that the measure is more important for intermediate levels of DebtRank and loses the explanatory power for extreme DebtRank levels. 

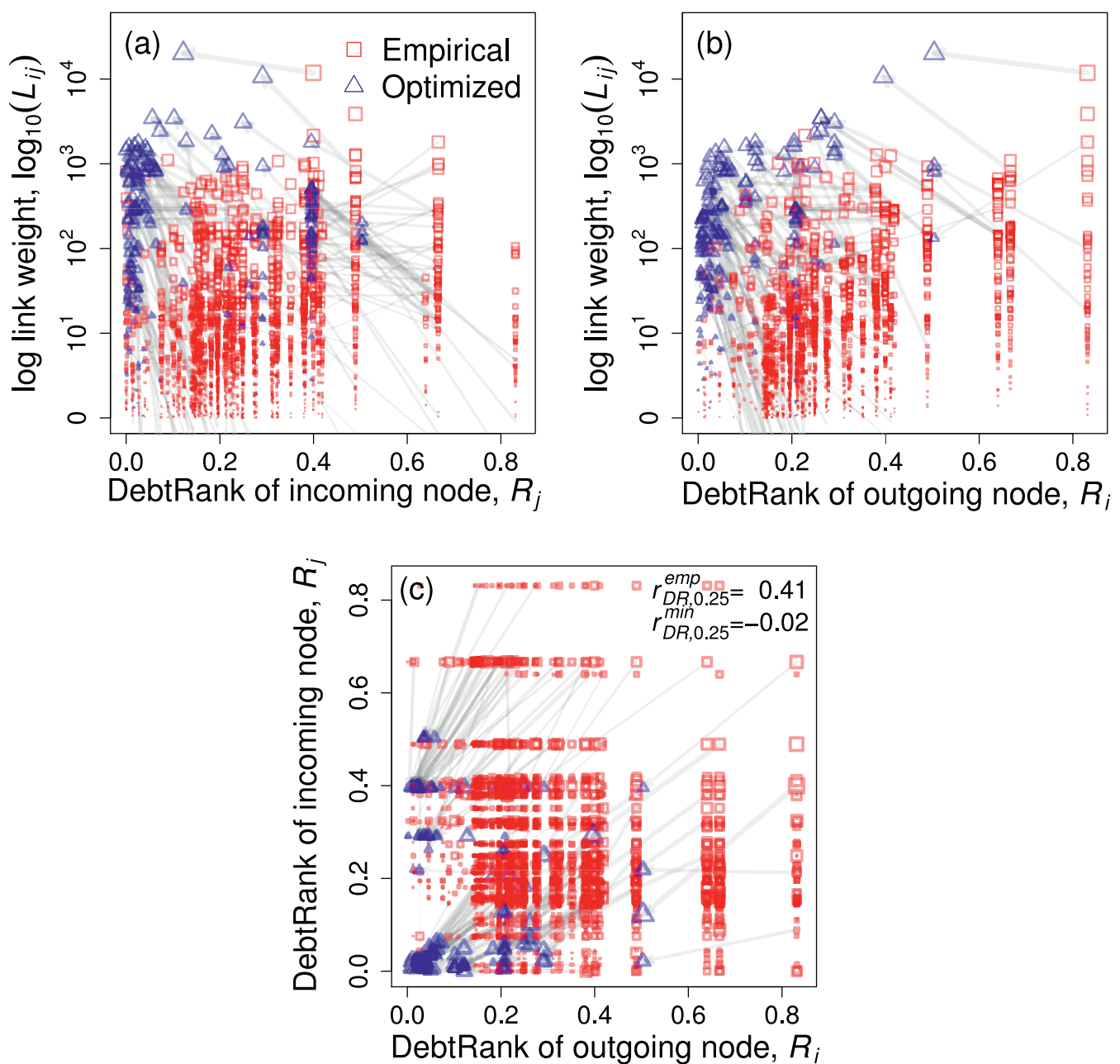

Fig. E.12. Link weights $L_{i j}$ and their relation to incoming $R_{j}$ and outgoing $R_{i}$ node DebtRank before (red squares) and after the optimization (blue triangles) for observation Q1. An arrow is drawn from an empirical link (red square) to an optimized link (blue triangle) if the link $L_{i j}>0$ in both networks. The size of the symbols (square, triangle, arrow width) corresponds to the respective link weight. (a) Shows the outgoing node DebtRank $R_{i}$ on the x-axes and $\log _{10}\left(L_{i} j\right)$ on the y-axes. We observe the general trend that link weights increase and the DebtRank of the outgoing node is reduced. In some rare cases link weights become smaller after the optimization (downward left arrows). Many small links in the empirical networks increase considerably in size. Some links retain their size but just move to the left, implying a reduction of DebtRank $R_{i}$ for the same link weight (horizontal left arrows). (b) Shows the incoming node DebtRank $R_{j}$ on the y-axes and $\log _{10}\left(L_{i} j\right)$ on the y-axes. Also in this case the general trend points to the top left. However, we see that in the center of the picture there is a cluster of blue triangles, which show decreases in link weights (arrows from top right to the center) and some increases in link weights (arrows from bottom right to the center). Interestingly, the optimization increases the weight of some the largest links in the empirical network. (c) For each link $L_{i j}$ we plot outgoing $R_{i}$ against incoming $R_{j}$ node DebtRank before (red squares) and after the optimization (blue triangles) for observation Q1. An arrow is drawn from an empirical link (red square) to an optimized link (blue triangle) if the link $L_{i j}>0$ in both networks. The size of the symbols (square, triangle, arrow width) corresponds to the respective link weight. The DebtRank weighted assortativity for the $25 \%$ banks with highest DebtRank is $r_{D R, 0.25}=0.41$ for the empirical network and $r_{D R, 0.25}=-0.02$ for the optimized network.

\section{Appendix F. DebtRank and link density for random networks}

Fig. 4 (c) shows that spare networks can have both very low and very high DebtRanks. As this relationship is a recurrent theme in the literature it is worthwhile exploring it further. The picture presented in Fig. 4(c) could either suggest that there is a strongly non-linear relationship in place or that the variance in DebtRank is considerably large for sparse networks. To investigate this relationship further, we conduct an exploratory simulation study by sampling 200,000 networks for each of the ten observations.

We employ the methodology developed by Gandy and Veraart (2016), which is conveniently available as R package ("systemicrisk"). One advantage of the methodology is that the algorithm samples networks with prespecified in- and outstrengths, allowing us to compare the samples meaningfully to the empirical and the optimized networks. We employ the additive exponential fitness model, which connects high fitness nodes with higher probability than nodes with low fitness. There are effectively five parameters to control, the expected out degree for banks with large and small fitness is controlled 

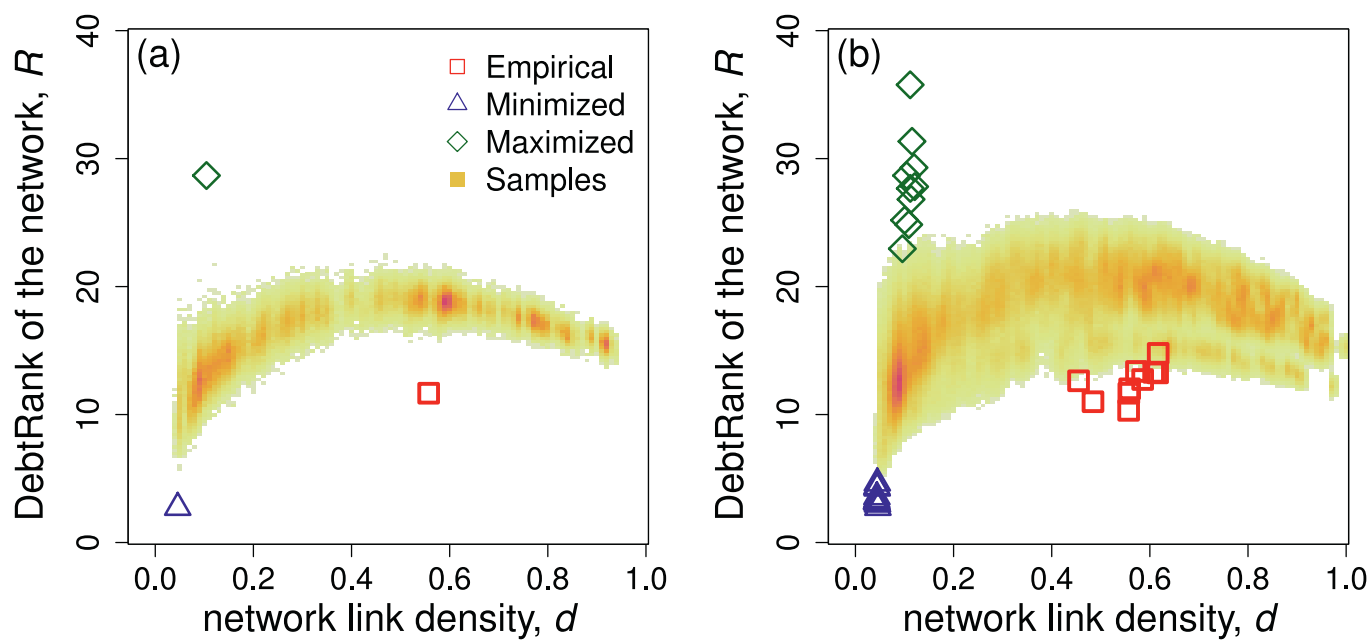

Fig. F.13. Extension of Fig. 4, which depicts the DebtRank on the y-axes and link density on the x-axes for the empirical (red squares), minimized (blue triangles), maximized (green diamonds), and randomly sampled networks (orange heat map; darker tones indicate that more sampled networks lie in this area). (a) Shows the relationship for observation Q8 and 200,000 randomly sampled networks with the same in- and out-strengths. The variance is visibly higher for sparse networks and decreases with link density. Additionally, a slightly concave shape seems to present, which peaks at medium density levels around 0.5 . The optimized and empirical networks lie outside the area covered by the sample. (b) Shows the same picture for all ten observations. The sample becomes more fragmented for higher density levels. The concave relationship is still visible. The higher variance on the sparse end is mostly disguised by the superposition of the ten different samples.

by $\gamma$ and $\beta$, the powerlaw exponent for the degree distribution is controlled by $\alpha$. The parameters $\zeta$ and $\eta$ control the power law exponent and the scale parameter of the link weight distribution (Pareto Type II distribution). For details, see Gandy and Veraart (2016). We initialize for each of the ten observations 100 runs where we sample the parameters $\gamma$ and $\beta$ uniformly between zero and one and the parameter $\alpha$ uniformly between minus two and minus four. The scale parameter $\eta$ is sampled exponentially with parameters 1000 , and the shape parameter $\zeta$ can be in the interval [1,2.5]. It should be pointed out that the small number of runs might not be sufficient to explore the entire parameter space. Moreover, we do not consider any alternative network sampling algorithms. Thus, the results have to be interpreted cautiously.

Nonetheless, the preliminary findings from our small scale simulation study - depicted in Fig. F.13 - are very interesting. (a) Shows the results for observations Q8. The optimization procedure achieves more extreme DebtRank levels than the simulations. The variance is considerably higher for networks with low link densities and potentially decreases with link density. Additionally, some of the highest and all of the lowest DebtRank values are observed for small densities. A possible presumption about the decreasing variance is that for almost fully connected networks with very equally distributed link weights there are only a smaller number of different network specifications possible, which are rather similar. Thirdly, the relationship is concave with high DebtRanks for intermediate levels of link density. Finally, the empirical network lies below the area covered by the sampled networks, indicating that the sample space was not explored extensively enough. This is an obvious limitation. (b) Shows the heatmap for the samples corresponding to the ten observations ${ }^{12}$ Across all quarters there is higher variance for sparse networks and the empirical networks lie below the sample area in all quarters. In general the samples differ by vertical shifts and different levels of concavity.

The sampling procedure can be seen as an indication that both patterns might be present: a higher variance of DebtRank for sparser networks and a concave non-linear relationship of DebtRank and link density. To corroborate the results more simulation studies should be conducted that use different sampling methods. In particular, comparing the results for algorithms generating different network topologies could shed more light on the relation between link density and systemic risk. The patterns identified above could be guiding questions for such future research.

\section{Appendix G. Comparing results with DebtRank2}

This section investigates the robustness of the proposed optimization with respect to the definition of DebtRank. For this reason we substitute the original DebtRank defined in Battiston et al. (2012c) by a variation that was proposed in Bardoscia et al. (2015), which we call DebtRank2. We find that the minimization procedure is still producing networks with lower systemic risk when DebtRank is interchanged with DebtRank2. However, there are differences. DebtRank2 $R^{2}$ is reduced on average by $15 \%$ from 59 to 50 . We present the corresponding plot to Fig. 1(a) and (b) in Fig. H.14. The first observation is

\footnotetext{
12 Joining the samples for all ten observations leads to the situation that some outliers, which are visible for a single observations, are not visible in the overall sample. This is because their relative frequency is decreased and the respective box is no longer colored (e.g., top left outliers).
} 

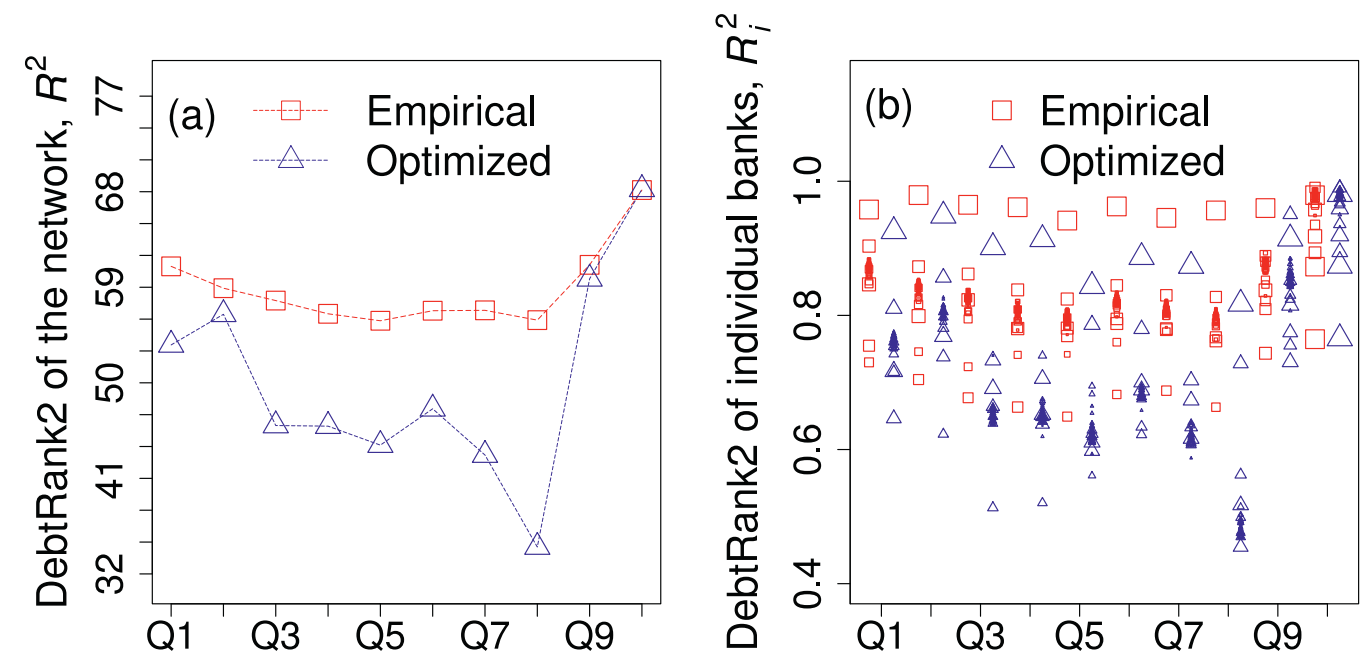

Fig. H.14. Same as Fig. 1(a) and (b) but for the definition of DebtRank2. In comparison to the ordinary DebtRank, we see that the DebtRank2 levels are substantially higher for both the empirical and minimized networks. In quarter Q10 both the empirical and the minimized DebtRank approach the theoretical maximum of DebtRank 69 for a network of 70 banks. The minimization works best for Q8 where the ratio of interbank market volume and equity $\bar{L} / \bar{E}$ is smallest. (b) shows that the optimization works better for smaller banks.

that the levels for DebtRank2 are substantially higher than for the original DebtRank. Additionally, we see that when the ratio $\bar{L} / \bar{E}$ increases, as shown in Fig. 1(c), the systemic risk reduction potential for DebtRank2 is diminishing in the last two observations. Furthermore, in H.14(b) we can observe that the single bank DebtRanks are more homogenous in comparison to Fig. 1(b).

\section{Appendix H. Estimating credit riskiness and data needs}

As in the empirical part of this paper, we focus on a direct exposure network, the specification of the credit risk variable, $\kappa$, needs to be considered. We suggest three state-of-the-art approaches to specify the credit riskiness of individual banks, which are found in McNeil et al. (2015). However, all of these have specific data needs, and to a large extent, this data is usually not publicly available. We therefore need to employ a proxy for $\kappa$ instead. The first and probably most broadly applicable choice for $\kappa$ are ratings, which are assigned to banks by rating agencies like Standard and Poors, Moodies and Fitch, which consider historical probabilities of default (PDs) for the respective rating class as $\kappa$. The disadvantage of this is that agencies usually update their ratings gradually and ratings are, to a certain extent, sticky. In suddenly deteriorating economic environments of financial crises, the ratings might not reflect the actual risk. The second approach suggested is using an analytical model, which can be calibrated to publicly available data. One of the most popular structural credit risk models is the Merton model Merton (1974). Several versions are used in practice to calculate PDs. The advantage of the model is that it can be calibrated with publicly available balance sheet data and stock market prices. The incorporation of stock market data captures most recent information. The third approach would be to consider CDS spreads of banks and infer the PDs from a reduced-form credit risk model, which is calibrated to the observed CDS spreads. In the reduced form approach the CDS spreads used to calibrate the model take into account current market information.

All of these approaches are generally difficult to put into practice as, for small banks, there might be insufficient information available to calibrate an analytical model. A possible solution could be to use credit risk estimates of banks, where data is available, and perform a regression analysis with accounting ratios as independent variables. The so fitted model could be used to obtain an estimate for the banks for which the necessary information is not accessible.

\section{Appendix I. Equality vs. inequality in the credit risk constraint}

$$
\begin{aligned}
\min _{L \in\left\{M \in \mathbb{R}_{+}^{N \times N}: \operatorname{diag}(L)=0\right\}} & \sum_{i=1}^{N} \sum_{j=1}^{N} \min \left(\frac{L_{i j}}{e_{j}}, 1\right) \frac{a_{j}}{\bar{L}} \\
\text { subject to: } & l_{i}=\sum_{j=1}^{n} L_{i j} \quad \forall i,
\end{aligned}
$$




$$
\begin{aligned}
& a_{i}=\sum_{j=1}^{N} L_{j i} \quad \forall i \\
& r_{i}=\sum_{j=1}^{N} L_{j i} \kappa_{j} .
\end{aligned}
$$

We show that in the formulation of the optimization problem (P1), changing the credit risk constraint (C3) from equality $(=)$ to greater or equal than $(\geq)$, does not change the solution, when the row and column sum constraints (C1) and (C2) are in place. This is intuitively clear because the row sum constraint keeps the amount of interbank liabilities in the system constant. This implies that the total sum of credit risk-weighted liabilities $\sum_{j=1}^{N} r_{j}=\sum_{j=1}^{N} \kappa_{j} l_{j}$ must also remain constant. If, now, one or more banks were to have a lower credit risk-weighted exposure $r_{i}$ after the optimization than before it and none of the banks had a higher risk weighted exposure $r_{j}$ (as implied by the $\geq$ constraint) this means that the total risk-weighted exposure $\sum_{j=1}^{N} r_{j}$ must be smaller after the optimization than before it, which is a contradiction of the statement that $\sum_{j=1}^{N} r_{j}$ must remain constant because the $l_{i}$ remain constant.

\section{References}

Acharya, V.V., Pedersen, L.H., Philippon, T., Richardson, M., 2017. Measuring systemic risk. Rev. Financ. Stud. 30 (1), 2-47. doi:10.1093/rfs/hhw088.

Adrian, T., Brunnermeier, M.K., 2016. CoVaR. Am. Econ. Rev. 106 (7), 1705-1741. doi:10.1257/aer.20120555.

Aldasoro, I., Gatti, D.D., Faia, E., 2017. Bank networks: contagion, systemic risk and prudential policy. J. Econ. Behav. Org. 142, 164-188. doi:10.1016/j.jebo. 2017.05.022.

Allen, F., Gale, D., 2000. Financial contagion. J. Polit. Econ. 108 (1), 1-33. doi:10.1086/262109.

Alter, A., Craig, B., Raupach, P., 2015. Centrality-based capital allocations. Int. J. Cent. Bank.

Artzner, P., Delbaen, F., Eber, J.-M., Heath, D., 1999. Coherent measures of risk. Math. Financ. 9 (3), 203-228. doi:10.1111/1467-9965.00068.

Bardoscia, M., Battiston, S., Caccioli, F., Caldarelli, G., 2015. Debtrank: a microscopic foundation for shock propagation. PLoS ONE 10 (6), e0130406. doi:10. 1371/journal.pone.0134888.

Bardoscia, M., Caccioli, F., Perotti, J.I., Vivaldo, G., Caldarelli, G., 2016. Distress propagation in complex networks: the case of non-linear debtrank. PLoS ONE 11 (10), e0163825. doi:10.1371/journal.pone.0163825.

Battiston, S., Gatti, D.D., Gallegati, M., Greenwald, B., Stiglitz, J.E., 2012. Default cascades: when does risk diversification increase stability? J. Financ. Stab. 8 (3), 138-149. doi:10.1016/j.jfs.2012.01.002.

Battiston, S., Gatti, D.D., Gallegati, M., Greenwald, B., Stiglitz, J.E., 2012. Liaisons dangereuses: increasing connectivity, risk sharing, and systemic risk. J. Econ. Dyn. Control 36 (8), 1121-1141. doi:10.1016/j.jedc.2012.04.001.

Battiston, S., Puliga, M., Kaushik, R., Tasca, P., Caldarelli, G., 2012. Debtrank: too central to fail? financial networks, the FED and systemic risk. Sci. Rep. 2 (541). doi:10.1038/srep00541.

BCBS, 2011a. Basel III: A Global Regulatory Framework for more Resilient Banks and Banking Systems Revised Version June 2011. Basel Committee on Banking Supervision.

BCBS, 2011b. Basel III Monitoring Report March 2019. Basel Committee on Banking Supervision.

BCBS, 2011. Global Systemically Important Banks: Assessment Methodology and the Additional Loss Absorbency Requirement. Basel Committee on Banking Supervision.

Beale, E.M.L., Tomlin, J.A., 1970. Special facilities in a general mathematical programming system for non- convex problems using ordered sets of variables. OR 69 (447-454), 99

Benoit, S., Colliard, J.-E., Hurlin, C., Pérignon, C., 2017. Where the risks lie: a survey on systemic risk. Rev. Financ. 21 (1), 109-152. doi:10.1093/rof/rfw026.

Biagini, F., Fouque, J.-P., Frittelli, M., Meyer-Brandis, T., 2018. A unified approach to systemic risk measures via acceptance sets. Math. Finan. doi:10.1111/ mafi.12170.

Boss, M., Elsinger, H., Summer, M., Thurner, S., 2004. Network topology of the interbank market. Quant. Financ. 4 (6), 677-684. doi:10.1080/ 14697680400020325.

Boss, M., Summer, M., Thurner, S., 2004. Contagion flow through banking networks. In: Proceedings of the International Conference on Computational Science. Springer, pp. 1070-1077. doi: 10.1007/978-3-540-24688-6_138.

Brownlees, C., Engle, R.F., 2016. SRISK: a conditional capital shortfall measure of systemic risk. Rev. Financ. Stud. 30 (1), 48-79. doi:10.1093/rfs/hhw060,

Caccioli, F., Farmer, J.D., Foti, N., Rockmore, D., 2015. Overlapping portfolios, contagion, and financial stability. J. Econ. Dyn. Control 51, 50-63. doi:10.1016/j. jedc.2014.09.041.

Cont, R., Moussa, A., Santos, E., 2010. Network structure and systemic risk in banking systems. SSRN doi:10.2139/ssrn.1733528.

Csardi, G., Nepusz, T., 2006. The igraph software package for complex network research. Int. J. Compl. Syst. 1695 (5), 1-9.

Eisenberg, L., Noe, T.H., 2001. Systemic risk in financial systems. Manag. Sci. 47 (2), 236-249. doi:10.1287/mnsc.47.2.236.9835.

Elsinger, H., Lehar, A., Summer, M., 2006. Risk assessment for banking systems. Manag. Sci. 52 (9), 1301-1314. doi:10.1287/mnsc.1060.0531.

Feinstein, Z., Rudloff, B., Weber, S., 2017. Measures of systemic risk. SIAM J. Financ. Math. 8 (1), 672-708. doi:10.1137/16M1066087.

Föllmer, H., Schied, A., 2002. Convex measures of risk and trading constraints. Financ. Stoch. 6 (4), 429-447. doi:10.1007/s007800200072.

Freixas, X., Parigi, B.M., Rochet, J.C., 2000. Systemic risk, interbank relations and liquidity provision by the central bank. J. Money Credit Bank. 32 (3), 611-638. doi:10.2307/2601198.

Gai, P., Kapadia, S., 2010. Contagion in financial networks. Proc. R. Soc. A: Math. Phys. Eng. Sci. 466 (2120), 2401-2423. doi:10.1098/rspa.2009.0410.

Gandy, A., Veraart, L.A., 2016. A Bayesian methodology for systemic risk assessment in financial networks. Manag. Sci. 63 (12), 4428-4446. doi:10.1287/ mnsc.2016.2546.

Gauthier, C., Lehar, A., Souissi, M., 2012. Macroprudential capital requirements and systemic risk. J. Financ. Intermed. 21 (4), 594-618. doi:10.1016/j.jfi.2012. 01.005.

Glasserman, P., Young, H.P., 2015. How likely is contagion in financial networks? J. Bank. Financ. 50, 383-399. doi:10.1016/j.jbankfin.2014.02.006.

Glasserman, P., Young, H.P., 2016. Contagion in financial networks. J. Econ. Lit. 54 (3), 779-831. doi:10.1257/jel.20151228.

Krause, S., Stefancic, H., Zlatic, V., Caldarelli, G., 2019. Controlling systemic risk - network structures that minimize it and node properties to calculate it. arXiv:1902.08483.

Leduc, M., Poledna, S., Thurner, S., 2017. Systemic risk management in financial networks with credit default swaps. SSRN doi:10.2139/ssrn.2713200.

Leduc, M.V., Thurner, S., 2017. Incentivizing resilience in financial networks. J. Econ. Dyn. Control 82, 44-66. doi:10.1016/j.jedc.2017.05.010.

Lehar, A., 2005. Measuring systemic risk: a risk management approach. J. Bank. Financ. 29 (10), 2577-2603. doi:10.1016/j.jbankfin.2004.09.007. 
León, C., Berndsen, R., Renneboog, L., 2014. Financial stability and interacting networks of financial institutions and market infrastructures. Eur. Bank. Cent. Discuss. Paper Ser. (2014-011) doi:10.2139/ssrn.2502832.

Markose, S., 2012. Systemic risk from global financial derivatives: a network analysis of contagion and its mitigation with super-spreader tax. Int. Monet. Fund (12-282).

Markose, S., Giansante, S., Shaghaghi, A.R., 2012. 'Too interconnected to fail' financial network of US CDS market: topological fragility and systemic risk. J. Econ. Behav. Org. 83 (3), 627-646. doi:10.1016/j.jebo.2012.05.016.

McNeil, A.J., Frey, R., Embrechts, P., 2015. Quantitative Risk Management: Concepts, Techniques and Tools. Princeton University Press.

Merton, R.C., 1974. On the pricing of corporate debt: the risk structure of interest rates. J. Financ. 29 (2), 449-470. doi:10.1111/j.1540-6261.1974.tb03058.x.

Molina-Borboa, J.L., Martınez-Jaramillo, S., López-Gallo, F., van der Leij, M., 2015. A multiplex network analysis of the mexican banking system: link persistence, overlap and waiting times. J. Netw. Theory Financ. 1 (1), 99-138. doi:10.21314/JNTF.2015.006.

Nier, E., Yang, J., Yorulmazer, T., Alentorn, A., 2007. Network models and financial stability. J. Econ. Dyn. Control 31 (6), $2033-2060$. doi:10.1016/j.jedc.2007. 01.014.

Pichler, A., Poledna, S., Thurner, S., 2020. Systemic risk-efficient asset allocation: minimization of systemic risk as a network optimization problem. J. Financ. Stab.

Poledna, S., Bochmann, O., Thurner, S., 2017. Basel III capital surcharges for G-SIBs are far less effective in managing systemic risk in comparison to networkbased, systemic risk-dependent financial transaction taxes. J. Econ. Dyn. Control 77, 230-246. doi:10.1016/j.jedc.2017.02.004.

Poledna, S., Martínez-Jaramillo, S., Caccioli, F., Thurner, S., 2018. Quantification of systemic risk from overlapping portfolios in the financial system. arXiv: 1802.00311.

Poledna, S., Molina-Borboa, J.L., Martínez-Jaramillo, S., van der Leij, M., Thurner, S., 2015. The multi-layer network nature of systemic risk and its implications for the costs of financial crises. J. Financ. Stab. 20, 70-81. doi:10.1016/j.jfs.2015.08.001.

Poledna, S., Thurner, S., 2016. Elimination of systemic risk in financial networks by means of a systemic risk transaction tax. Quant. Financ. 16 (10), 15991613. doi:10.1080/14697688.2016.1156146.

Puhr, C., Seliger, R., Sigmund, M., 2012. Contagiousness and vulnerability in the Austrian interbank market. Oesterreichisch. Natl. Bank Financ. Stab. Rep. 24.

Theußl, S., Schwendinger, F., Hornik, K., 2019. ROI: an extensible R optimization infrastructure. Research Report Series Department of Statistics and Mathematics, 133. WU Vienna University of Economics and Business.

Thurner, S., Hanel, R., Klimek, P., 2018. Introduction to the Theory of Complex Systems. Oxford University Press.

Thurner, S., Poledna, S., 2013. Debtrank-transparency: controlling systemic risk in financial networks. Sci. Rep. 3, 1888. doi:10.1038/srep01888. 